\title{
Simultaneous retrieval of optical gains, losses, and threshold in active waveguides
}

\author{
Luis Cerdán* \\ Affiliation until 2017 \\ Instituto de Química Física "Rocasolano" (IQFR), Consejo Superior de Investigaciones Científicas \\ (CSIC), Serrano 119, 28006, Madrid, Spain \\ E-mail: lcerdanphd@gmail.com
}

The generation of Amplified Spontaneous Emission (ASE) is routinely used to assess the performance of active materials prior to developing thin film lasers. The Variable Stripe Length method has been profusely used for decades to measure the optical gain in these materials and devices, but experimental and theoretical evidences cast doubts upon its reliability. With the advent of an ever increasing number of solution-processed laser compounds that can be implemented as low-cost, flexible, and/or integrated devices, there is now more than ever a need to develop an alternative and trustful method to delineate all their amplifying signatures. Herein, a new formalism to fully characterize the ASE properties, based on the most frequent Variable Pump Intensity method, is reported. An analytical expression taking into account the most relevant spatiotemporal and photophysical effects has been found that can be fitted to ASE spectra. This expression allows retrieving, from a single ASE experiment, the spectrally resolved losses coefficient and net optical gains at all pump values, as well as to calculate, instead of visually finding, the ASE threshold. The reported formalism serves as a tool to categorize the best candidates for a given laser application through a reliable, robust, time-saver, and standardized methodology.

Keywords: laser physics; gain materials; waveguides; amplified spontaneous emission; graphical user interface 


\section{Introduction}

Since the invention of the laser by Theodor Maiman in 1960, the development of new laser materials has remained a hot research topic lying in the frontier between laser and materials sciences. In the last decades there has been a renewed interest in this field thanks to the discovery and development of a myriad of new organic,,$^{[1,2]}$ inorganic, ${ }^{[3-5]}$ and hybrid ${ }^{[6-8]}$ solution-processed laser compounds that can be implemented as waveguiding devices. ${ }^{[9,}{ }^{10]}$ Emitting across the whole visible spectrum and even the UV and IR, these materials outstand by their huge processing and functionalization versatility and for being ideally suited for the implementation of low-cost, flexible, and/or integrated lasers. ${ }^{[1,6,9-11]}$

With such a profusion of novel laser compounds, there has always been a need to develop characterization techniques to delineate or establish figure of merits of their amplification properties (i.e., optical gain and losses) that allow a performance comparison and help selecting or distinguishing the best candidates for a given laser application. Although the use of the wellestablished and powerful time-resolved femtosecond pump-probe spectroscopy technique enables the access to very useful information on the spectroscopic parameters and dynamics associated to both the ground and excited states, ${ }^{[12]}$ the methodologies based on the measurement of Amplified Spontaneous Emission (ASE) are preferred for the study of active materials for waveguide lasers. The reason for this choice lays not only in the simplicity of both its experimental implementation and its subsequent data analysis, but also because they are conducted at the pumping geometry and high intensity irradiation conditions required to achieve laser emission. In addition, these measurements are performed directly onto the waveguide device, implying that they naturally incorporate wave-guiding effects, such as photonic confinement and modal and scattering losses, regardless of how complicated its waveguide structure is. And last but not least, any material displaying ASE could emit laser radiation provided an adequate resonant cavity were implemented. Hence, the presence of ASE serves as an indicator of the potential of a given material to perform as a laser. 
In ASE experiments, the sample or waveguide is transversally pumped by a laser beam spatially conformed as a stripe-shape spot whose length is much longer than its width and whose end is placed right up to the edge of the film. The edge-emitted ASE is detected and measured as a function of certain experimental conditions (Figure 1). Based on this simple pump/detection geometry three different but complementary experiments can be performed.

The waveguide losses coefficient $\alpha$ can be obtained by using the Shifting Excitation Spot (SES) method, ${ }^{[13]}$ which consists of exciting the sample with a constant stripe length and pump value and gradually shifting it away from the edge of the sample (Figure 1b). The detected ASE decreases according to the Lambert-Beer's law, $I_{\text {out }} \propto \exp (-\alpha x)$, where $x$ is the distance between the end of the pump stripe and the edge of the sample. This method is highly useful but can lead to misleading results due to artefacts arising from "wrong coupling" effects in the detection system. ${ }^{[14]}$

a)

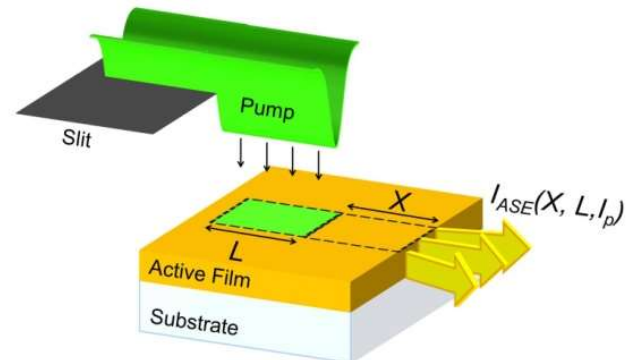

b)

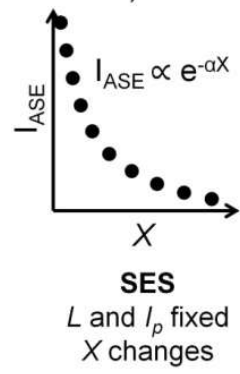

c)

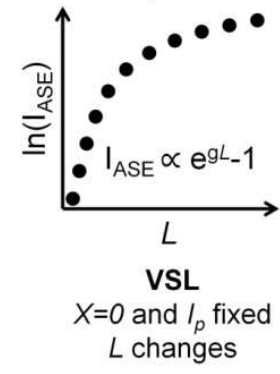

d)

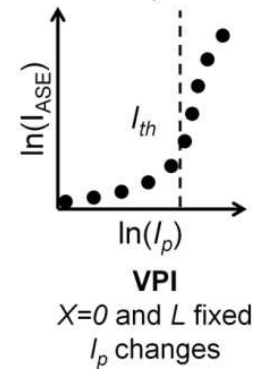

Figure 1: a) Sketch of different pumping geometries used to measure ASE properties in active waveguides. Qualitative representation of the variation of the ASE peak intensity as a function of: b) distance to edge $X$ (SES method), c) stripe length $L$ (VSL method) and d) pump value (VPI method).

The Variable Stripe Length (VSL) method is a very popular tool to measure the optical gain in thin film active devices. Basically, the intensity of the edge-emitted ASE is measured as a function 
of the excitation stripe length at fixed pump intensity (Figure 1c). The growth of ASE intensity $\left(I_{A S E}\right)$ as a function of the stripe length when gain saturation is neglected (small-signal approximation) follows the well-known expression: ${ }^{[15,16]}$

$$
I_{A S E}=\frac{\Omega \eta}{g}\left(e^{g L}-1\right)
$$

where $L$ is the stripe length, $g$ is the net optical gain coefficient, $\eta$ is the population in the excited state, and $\Omega$ is a proportionality parameter that contains information about the photophysical and geometrical properties of the active thin film. There are alternative analytical expressions to retrieve gains from VSL measurements, ${ }^{[17-21]}$ but equation (1) is the most frequently used one. A simple fit of equation (1) to the experimental $I_{A S E}$ vs. $L$ values gives the net optical gain as an output parameter. In addition, the VSL method can be used as an alternative to the SES method to estimate the waveguide losses coefficient $\alpha$, since at very low pump intensities $g \rightarrow-\alpha \cdot{ }^{[22]}$ However, over the last two decades, experimental and theoretical evidence has been reported that casts doubts upon its reliability and that seriously discourages its application (see ref. [23] and references therein). The most critical flaw arises due to the fact that the "small-signal" gain approximation used to derive equation (1) is rather coarse and leads to unreliable results, since the excited state population (and in turn the gain) becomes saturated and inhomogeneous along the excited region even at the shortest stripe lengths. ${ }^{[24]}$ A direct consequence of the oversimplified equation (1) is that the particular stripe length range chosen to perform the VSL fit has a profound effect on the final gain, as I have recently reported. ${ }^{[23]}$ In fact, a single set of VSL data may render gain values that differ by tens of $\mathrm{cm}^{-1}$.

Finally, in the Variable Pump Intensity (VPI) method, which is by far much more frequently used than both the SES and VSL methods, the output ASE intensity is acquired as a function of the pump excitation value (energy, density, intensity, ...) keeping the stripe length fixed (Figure 1d). From this experiment, which is completely analogous to that performed for lasers, one usually retrieves, upon visual inspection, both the ASE threshold pump value and peak wavelength. The 
ASE threshold is one of the most important ASE parameters, as it is commonly used as a figure of merit for the goodness of a given compound as a laser material. ${ }^{[1]}$ One of the advantages of the VPI method is that, unlike the SES and VSL ones, it does not suffer from detection coupling artefacts, as pump and detection geometries are kept untouched. Unfortunately, there is not a reliable way of obtaining specific information on the optical gain of the sample. In other words, nobody has found a solid and trustful expression analogous to equation (1) but that can be applied to the VPI experiment. Silvestre et al. reported a formalism to retrieve optical gains from VPI measurements, ${ }^{[25]}$ but their method has not gained much traction, probably because their assumptions were not too realistic. ${ }^{[26,27]}$

As we have seen, up to three analogous but independent experiments (SES, VSL, VPI) are currently required to fully characterize the ASE properties of active thin films (losses, gains, and threshold). As the three methods are based exactly on the same physical principles and pump and detection geometries (Figure 1), logic dictates that there must be some way of obtaining all that ASE information from a single experiment. Remarkably, none has succeeded in solving this intriguing problem that would have far reaching implications for the scientific community working in laser materials and devices, as it would reduce the amount of experimental workload needed to fully characterize new samples (1 experiment vs. 3 experiments) and would contribute to standardize this kind of measurements and characterizations.

With this conundrum in mind, in this contribution I embark in an ambitious but successful attempt to derive a sufficiently simple analytical expression taking into account relevant gain and mode propagation processes that enables obtaining as much spectroscopic information on the ASE parameters as possible from active waveguides using exclusively the VPI method. Following a reductionist approach aided by numerical simulations, I have found a manageable analytical expression to describe the growth of the ASE intensity as a function of the pump value that includes implicitly the spatiotemporal variation of gain as well as gain and pump saturation effects. This new expression is a generalization of that of the VSL method (equation 1) and opens the door to 
simultaneously retrieve, from a single VPI experiment, the losses coefficient and the net optical gains at all pump values of the experiment with spectral resolution. In addition, thanks to a well defined objective criterion, the new formalism enables calculating, instead of visually finding, the ASE thresholds.

\section{Fundamentals and implementation}

\subsection{Analytical expression for the VPI method}

The main goal of this paper is to find an analytical expression from the whole set of spectrally resolved rate equations describing the spatiotemporal dynamics of ASE generation and propagation in waveguides or solutions ${ }^{[28]}$ (equations (A1)-(A3)) that can be fitted to the experimental ASE spectra. Of course, there is no way of exactly solving that system of coupled non-linear equations and thus a number of assumptions must be done and adequate approximations must be applied. The reductionist approach that I have followed entails a lengthy and cumbersome derivation involving numerical simulations whose full description does not help in the manuscript readability. The interested reader can find the details of this derivation in Appendix A. In this section I will just summarize the main findings.

Unfortunately, to derive a sufficiently simple analytical solution, one must sacrifice altogether the explicit spatial variation of the population inversion and use instead spatial averages. In other words, one must assume a homogeneous gain distribution. Once this coarse approximation is applied, it can be shown that the analytical expression describing the growth of ASE intensity $I_{A S E}\left(\lambda, I_{p}, L\right)$ as a function of the wavelength, pump value $\left(I_{p}\right.$; energy/density/intensity/flux/...) and stripe length $(L)$ reads:

$$
I_{A S E}\left(\lambda, I_{p}, L\right)=\frac{\Omega(\lambda) \eta\left(I_{p}\right)}{\gamma(\lambda) \Delta n\left(I_{p}\right)-\alpha(\lambda)}\left(e^{\left(\gamma(\lambda) \Delta n\left(I_{p}\right)-\alpha(\lambda)\right) L}-1\right)
$$

with 


$$
\Delta n\left(I_{p}\right)=\Delta n_{0} \frac{\frac{I_{p}}{I_{p, 1}^{s}}+\left(\frac{I_{p}}{I_{p, 2}^{s}}\right)^{m}}{1+\frac{I_{p}}{I_{p, 1}^{s}}+\left(\frac{I_{p}}{I_{p, 2}^{s}}\right)^{n}},
$$

Equations (2a) and (2b) represent the main results of this manuscript and will be the expressions to be used for the fitting procedure in the following sections. It is easy to see that equation (2a) is a generalization of the common VSL expression equation (1) that has been used for decades. The functions $\eta$ and $\Delta n$ represent different measures of the temporally integrated spatially averaged excited state population. Nevertheless, the so-called effective population inversion $\Delta n$ quantifies how well the actual population inversion and the photon flux overlap in time. In equation $(2 \mathrm{~b}), \Delta n_{0}$ is a proportionality factor, $I_{p, 1}^{s}$ and $I_{p, 2}^{s}$ are a sort of saturation intensities, and $m$ and $n$, with $m>n$, are exponents that determine how fast or slow the gain grows and saturates. This equation fulfils the fundamental condition imposing that $\Delta n \rightarrow 0$ as $I_{p} \rightarrow 0$. It is enticing to relate $I_{p, 1}^{s}$ and $I_{p, 2}^{s}$ with the pump and gain saturation intensities, respectively, but the evidences don't seem to support this relation. Table 1 summarizes all the spatiotemporal, photophysical and saturation processes implicitly taken into account in equation (2b). It must be highlighted that this expression even takes into account singlet-singlet annihilation, an energy transfer process that it is known to reduce the laser efficiency. ${ }^{[6]}$

In equation (2a), the fluorescence parameter $\Omega(\lambda)=\Omega_{0} \cdot \mathrm{F}(\lambda)$ contains information about the radiative properties of the active medium and its fluorescence spectrum $F(\lambda)$ as measured in a spectrofluorimeter (equation (A4h)). The total losses coefficient $\alpha(\lambda)$ accounts for the ground state absorption (GSA) and scattering/modal (SC) coefficients as:

$$
\alpha=\alpha(\lambda)=\Gamma(\lambda)\left(\alpha_{S C}(\lambda)+\alpha_{G S A}(\lambda)\right)
$$

where the mode confinement function $\Gamma(\lambda)$ garners all information on the spatial overlap between the pump value variation across the film and the mode profile, ${ }^{[29-31]}$ as well as information on all 
Table 1. Spectral, spatiotemporal and gain saturation assumptions taken into account to derive the analytic expressions for the VSL and VPI methods as compare to real life.

\begin{tabular}{ccc}
\hline Assumption & VPI method (Equations (2)) & $\begin{array}{c}\text { VSL method } \\
\text { (Equation (1)) }\end{array}$ \\
\hline Polychromatic & $\checkmark$ & $\square$ \\
Temporal evolution & $\checkmark($ Implicit in $\Delta n)$ & $\square(\mathrm{CW})$ \\
Bidirectional photon flux & $\checkmark($ Implicit in $\Delta n)$ & $\checkmark$ \\
Spatial variation of photon flux & $\checkmark$ & $\square$ \\
Spatial variation of population inversion & $\checkmark($ Implicit in $\Delta n)$ & $\square$ \\
Pump Saturation & $\checkmark($ Implicit in $\Delta n)$ & $\square$ \\
Gain Saturation & $\checkmark($ Implicit in $\Delta n)$ & $\square$ \\
Singlet-singlet annihilation & $\checkmark($ Implicit in $\Delta n)$ & $\checkmark($ Implicit in g) \\
Stimulated emission & $\checkmark($ Implicit in $\gamma)$ & $\checkmark($ Implicit in g) \\
Excited State Absorption & $\checkmark($ Implicit in $\gamma)$ & $\checkmark($ Implicit in g) \\
Ground State Absorption & $\checkmark($ Implicit in $\gamma$ and $\alpha)$ & $\checkmark($ Implicit in g) \\
Modal Losses & $\checkmark($ Implicit in $\alpha)$ & \\
\hline
\end{tabular}

possible propagation and interference effects (e.g., leaky-modes $\left.{ }^{[32]}\right)$. The gross optical gain coefficient $\gamma(\lambda)$ in equation (2a) is given by:

$$
\gamma(\lambda)=\xi(\lambda) \Gamma(\lambda)\left(\alpha_{S T}(\lambda)+\alpha_{G S A}(\lambda)-\alpha_{E S A}(\lambda)\right)
$$

where $\alpha_{S T}(\lambda), \alpha_{G S A}(\lambda)$ and $\alpha_{E S A}(\lambda)$ are, respectively, the stimulated emission (ST), ground state absorption (GSA) and excited state absorption (ESA) coefficients. It is of utmost importance to note that with the approximations and assumptions carried out in the present formalism (which are the same as those underlying the VSL method) it is unveiled that the measured optical gross gain is not the sum of the ST and GSA coefficients minus the ESA one, multiplied by the mode confinement function. ${ }^{[31,33,34]}$ Instead, it is spectrally corrected by $\xi(\lambda)$ (Figure A4a), a parameter, normalized so that $\int \xi(\lambda) d \lambda=1$, that takes into account the differences in amplification strength as a function of wavelength stemming from the combination of two facts: 1) the differences in temporal overlap between the excited state population and the photon flux at each wavelength, and 2) the assumption that both $\eta$ and $\Delta \eta$ are spatially homogenous. Obviously, under CW excitation $\xi(\lambda)$ would lose its spectral dependency and would become a constant (cf. equations (A13b) and (A16)). In any case, 
the correction $\xi(\lambda)$ results in an overestimation of the gross optical gain at the spectral edges with respect to the real ones. Fortunately, it is at the edges of the experimental spectra where the signal to noise ratio is smaller and thus the fit errors associated to it will compete with those of the overestimation. Nevertheless, both effects have to be taken into account when interpreting the results of the fit. Finally, the net optical gain $g$ is defined as:

$$
g=g\left(\lambda, I_{p}\right)=\gamma(\lambda) \Delta n\left(I_{p}\right)-\alpha(\lambda)
$$

As a final comment, although it has not been included in the notation of equations (2)-(5), both $\eta$, $\Delta n, \xi(\lambda)$, and, consequently, $\gamma(\lambda)$, depend implicitly on the stripe length $L$. This hidden dependency basically implies that a change in the latter would result in a change in the formers. In practical terms, if two or more samples are to be compared using the VPI method, both experiments must be performed with the same stripe lengths.

\subsection{ASE Threshold}

Up to date, the most important parameter extracted from a VPI experiment has been the threshold, as it is commonly used as a figure of merit for the goodness of a given compound as a laser material. Thus, an accurate determination of this parameter is essential. Most criteria to find the threshold rely on the visual inspection of changes in a given emission parameter. The most frequently used method consists of localizing the pump intensity at which there is a change in slope in the input-output intensity plot (Figure 1d and refs. [5,7,22,35]). Other authors consider that the ASE threshold lays at the pump intensity at which the Full Width Half Maximum (FWHM) reaches half its value at the lowest pump intensities. ${ }^{[36-38]}$ In yet another few publications, it is assumed to be reached previously, where the ASE FWHM just begins to narrow. ${ }^{[39]}$ A different approach, exactly analogous to that of the laser threshold, considers the threshold intensity to be that for which the gross gain compensates the losses, i.e., that which nulls the net optical gain equation (5). ${ }^{[40]}$ In a previous paper, my co-workers and I applied a criterion that reads: "The threshold condition for ASE is realized when the stimulated and the spontaneously emitted photon fluxes are, after losses, equal at the edge of the excitation region. In other words, at threshold the ASE output intensity must 
be twice the fluorescence [one]."[41] This last criterion, which is most accurate, will be used again herein. In this case, the ASE output intensity $I_{A S E}$ is given by Equation (2a), while the fluorescence intensity $I_{F}$ is given as well by Equation (2a) but assuming that there is no gain whatsoever, i.e., setting $\gamma=0$. Thus, the threshold condition $I_{A S E}=2 I_{F}$ at the ASE peak wavelength reads:

$$
\frac{\left(e^{\left(\gamma\left(\lambda_{A S E}\right) \Delta n\left(I_{p, t h}\right)-\alpha\left(\lambda_{A S E}\right)\right) L}-1\right)}{\gamma\left(\lambda_{A S E}\right) \Delta n\left(I_{p, t h}\right)-\alpha\left(\lambda_{A S E}\right)}=2 \frac{\left(1-e^{-\alpha\left(\lambda_{A S E}\right) L}\right)}{\alpha\left(\lambda_{A S E}\right)}
$$

Once $\gamma\left(\lambda_{\mathrm{ASE}}\right), \Delta n\left(I_{p}\right)$, and $\alpha\left(\lambda_{\mathrm{ASE}}\right)$ are known, one can calculate the threshold by finding the pump value $I_{p}=I_{p, t h}$ fulfilling equation (6). The main advantages of using a criterion that allows calculating, instead of guessing, the ASE threshold are, first, the prevention of errors arising from subjective visual estimations and, second, the standardization of the characterization of laser materials. One may wonder how the ASE threshold obtained using the criterion described by equation (6) compares to those obtained visually (i.e., change in $I_{A S E}$ slope, FWHM/2, FWHM narrowing, ...). After analysing several cases, the conclusion is that the exact point will vary from sample to sample and will ultimately depend on the particular values of the gross gain $\gamma$, the total losses $\alpha$, and the exact shape of $\Delta n$. But in general, the computed value usually lies close to where the change in slope in the input-output intensity plot takes place, which is most fortunate given that that is the most frequently used criterion in the literature.

\subsection{Implementation of VPI method}

\subsubsection{Fitting procedure}

The particular fitting procedure to apply the VPI method involves many fitting parameters and is very sensitive to initial conditions. Consequently, a number of constraints have to be included and the fit must be performed in a sequential fashion. The detailed aspects of my recipe to fit equations (2a) and (2b) to the experimental ASE spectra are described in the Supplementary Section S1. In this section I will include some important details.

To start with, $\gamma(\lambda), \eta\left(I_{p}\right)$ and the parameters associated to $\Delta n\left(m, n, I_{p, 1}^{s}\right.$ and $\left.I_{p, 2}^{s}\right)$ can be left free. The fluorescence spectrum $F(\lambda)$, as measured in a spectrofluorimeter, is used as a constraint, and the 
losses $\alpha(\lambda)$ are estimated, in a first step, as follows. In the limit when $I_{p} \rightarrow 0$, equation (2) can be approximated to:

$$
I_{A S E}\left(L, I_{p} \rightarrow 0, \lambda\right) \propto \frac{F(\lambda) I_{p}}{\alpha(\lambda)}\left(1-e^{-\alpha(\lambda) L}\right)
$$

Thus, one can retrieve an estimation of $\alpha(\lambda)$ fitting eq. (7) to the measured ASE spectra at pump intensities well-below threshold. Using all the ASE spectra in subsequent fits, this estimation is refined and the rest of parameters are obtained. Furthermore, the inspection of equations (2a), (2b), and (A4h) reveals that $\Omega_{0}$ and $\eta$, and $\gamma$ and $\Delta n_{0}$, appear always as a product, i.e., $\Omega_{0} \eta$ and $\gamma \Delta n_{0}$. Whatever the fitting method, in the process of inspecting the parameter space to optimize the fit, an infinite number of combinations would be found such that the product of the fitted parameters is equal to the product of the actual parameters $\left(e . g ., \Omega_{0, \text { fit } 1} \eta_{\mathrm{fit} 1}=\Omega_{0, \mathrm{fit} 2} \eta_{\mathrm{fit} 2}=\ldots=\Omega_{0} \eta\right)$. This leads to a huge uncertainty and makes the determination of errors impossible. To avoid this, I have found convenient to normalize $\Delta n$ and $\eta$ to unity at the maximum pump intensity of the experiment $\left(I_{p, \max }\right)$. The proportionality factors that are cancelled out in the normalization of $\Delta n$ and $\eta$ are assimilated into $\gamma(\lambda)$ and $\Omega_{0}$, respectively. This has a trade-off in that the fitted $\gamma$ will be, in general, not absolute anymore, but relative. In contrast, the product $\gamma \Delta n$ will be absolute. This has to be taken into account when analysing the results of the fit.

\subsubsection{Graphical User Interface (GUI): ASE gain Labs (AgL)}

The described fitting procedure may not be trivially implemented. Therefore, I have developed the stand-alone GUI "ASE gain Labs" $(A g L)$ to perform this task automatically. The user must provide the value for the stripe length $L$, and the files with the fluorescence spectrum $\mathrm{F}(\lambda)$ (acquired in a spectrofluorimeter in front-face configuration), the ASE spectra, and the pump values $I_{p}$ in whatever units the user considers (energy, intensity, fluence, power, flux, ...). The GUI can be obtained from the author at no cost and can be freely distributed. The GUI runs on Microsoft Windows operating systems. It has been checked to run in Windows XP, Windows Vista, Windows 7, and Windows 10. 


\section{Proof of concept and benchmarking}

\subsection{Experimental case study}

Prior to analyse an experimental case study, the performance and sensitivity to noise of the VPI method and the GUI $\operatorname{AgL}$ was assessed under the ideal and controlled case corresponding to ASE spectra generated numerically (see Supplementary Section S2). The fitting parameters almost mimicked to perfection the ideal numerical results (Figure S1) and were reasonably immune to the presence of noise even up to a $30 \%$ error (Figure S2). This analysis bears witness of the reliability and robustness against noise of the presented methodology and the developed software.

To check its reliability and performance under real experimental conditions, the VPI method presented in the foregoing sections was tested, through the use of the GUI $A g L$, against ASE spectra from several dye doped polymer thin films measured in the past in our labs. ${ }^{[40,42-47]}$ In addition, to avoid a bias towards our lab results, the method and GUI $A g L$ were tested against ASE spectra obtained from several samples measured in the labs of independent researchers not related with the author. In particular, these latter samples were based on carbon-bridged oligo(pphenylenevinylene)s, ${ }^{[48]}$ benzo[c]fluorene core molecular glasses, ${ }^{[49]}$ and poly $(9,9$-dioctylfluorene) (PFO). ${ }^{[29]}$ A total of more than 40 different data sets were analysed and the results were highly successful and satisfactory. Some of these case studies will be evaluated in a future publication for their relevance in probing the limits of this formalism. Remarkably, the GUI $A g L$ behaved strikingly well considering that none of the analysed data sets were measured with this method in mind. The performance against real experimental results highlights the relevance, power, reliability and robustness of both the VPI method and the GUI $A g L$. 

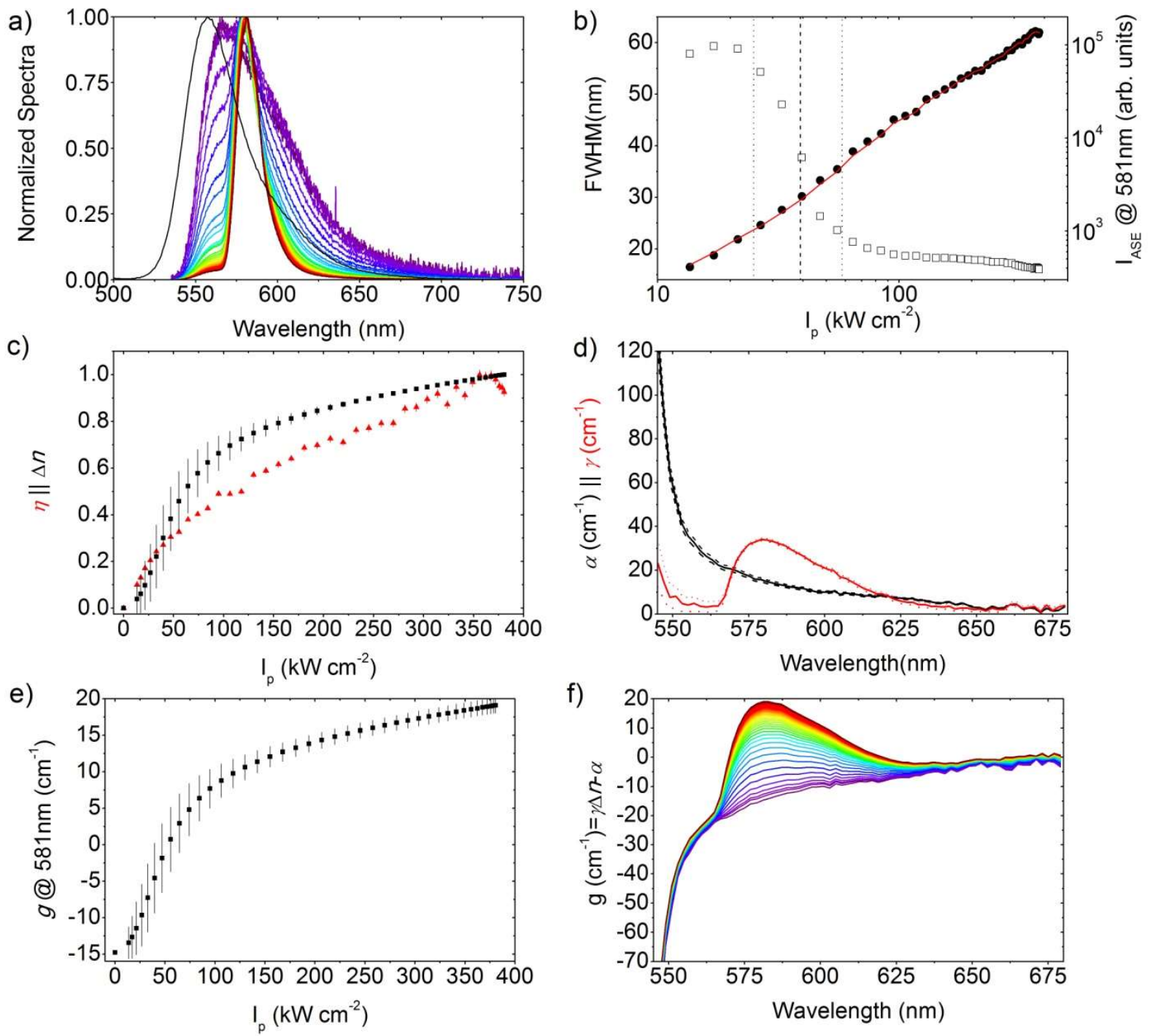

Figure 2: Analysis of sample Rh6G/pHEMA with the VPI method and GUI $A g L$ : a) Normalized fluorescence and ASE spectra used for the fit. b) ASE intensity at the peak wavelength $(581 \mathrm{~nm})$ and ASE full width half maximum (FWHM) as a function of pump intensity. The vertical dashed and dotted lines mark the position, respectively, of the ASE threshold calculated with equation (6) and its corresponding 95\% confidence interval. The red solid line is the best fit to the experimental values. c) Fitted function $\eta$ and effective population inversion $\Delta n$ as a function of pump intensity. d) Fitted losses and gross gain as a function of wavelength. e) Fitted net optical gain at ASE peak as a function of pump intensity. f) Spectrally resolved net optical gain at increasing pump intensities.

In what follows I will present the results of the fit corresponding to a representative example: an asymmetric slab waveguide consisting of a 1 micron thick $10 \mathrm{mM}$ Rhodamine $6 \mathrm{G}(\mathrm{Rh} 6 \mathrm{G})$ doped pHEMA film deposited onto a quartz substrate (Details on experimental conditions in ref. [44]). The fluorescence and ASE spectra (Figure 2a) together with the pump intensity values were loaded onto the GUI $A g L$. The fit was performed between $545 \mathrm{~nm}$ and $680 \mathrm{~nm}$ with a wavelength sampling $\Delta \lambda=2 \mathrm{~nm}$ and a stripe length $L=0.2 \mathrm{~cm}$. For the current data set there was already a slight 
evidence of amplification at the second lowest pump intensity (Figure 2b) and, hence, only the spectrum corresponding to the lowest pump intensity was used to retrieve the losses. For the full fit, all the available ASE spectra (41 in this data set) were used. Under these conditions, the fit took slightly above 1 minute to finish in an old Toshiba laptop with an Intel ${ }^{\circledR}$ Core 2 duo P8700 (2.53 $\mathrm{GHz}$ ) processor. The fit to the experimental data had a good level of goodness, as can be testified by the similarity between the fitted and experimental ASE peak intensities (Figure 2b), as well as by the small fitting errors in $\eta$ and $\Delta n$ (Figure 2c), and in the losses and gross gain spectra (Figure $2 \mathrm{~d}$ ).

The ASE pump threshold $\left(I_{p, t h}\right)$ of this sample was originally determined visually finding the pump intensity at which there was a change in slope in the input-output intensity plot, and was placed close to 30 or $40 \mathrm{~kW} \mathrm{~cm}^{-2}$. The exact point was difficult to locate since the change in slope was very diffuse (Figure 2b). With the current VPI method and thanks to the threshold criterion given by equation (6), the ASE threshold has been calculated to be at $39 \mathrm{~kW} \mathrm{~cm}^{-2}$ (Figure $2 \mathrm{~b}$ ), which is very close to the visually estimated one. Another advantage of using an analytical expression to calculate, instead of guessing, the threshold, is that it allows obtaining its associated uncertainties through the use of error propagation treatments. In this sample, the ASE threshold can be found with a $95 \%$ confidence level in the interval $I_{p, t h} \in[25,58] \mathrm{kW} \mathrm{cm}^{-2}$ (Figure $2 \mathrm{~b}$ ). This uncertainty could be reduced using a finer wavelength sampling $\Delta \lambda$, but that would increase the fitting time.

The function $\eta$ and the effective population inversion $\Delta n$ follow a similar dependency on the pump intensity (Figure 2c) as both parameters have in their cores the real population inversion (cf. equations (A13), and (A16)). They differ primarily due to $\tau_{i}$, the function acting upon $n_{2}$ (cf. equation (A13b)) which makes $\Delta \eta$ taking into account the temporal overlap between the population inversion $n_{2}$ and the photon flux.

The fitted losses coefficient spectrum $\alpha$ (Figure $2 \mathrm{~d}$ ) has a shape closely resembling an exponential decay comprised of the long wavelength tail of the absorption band and the scattering/modal losses. ${ }^{[50]}$ The losses at the ASE peak $\left(\alpha\left(\lambda_{\mathrm{ASE}}\right)=14.8 \pm 0.8 \mathrm{~cm}^{-1}\right)$ are of the order 
of magnitude of those found in other dye doped polymer waveguides, ${ }^{[21,22,38,40,45,50]}$ and consistent with the extinction coefficient expected for Rh6G $10 \mathrm{mM}$ at $580 \mathrm{~nm}$. Note that with the VPI method, time has been saved by avoiding the need to perform an independent experiment to estimate the waveguide losses spectrum applying the SES method (Figure 1b).

On the other hand, the shape of the fitted gross gain spectrum $\gamma$ (Figure $2 \mathrm{~d}$ ) is consistent with that expected from the sum of the ground state absorption (GSA) and stimulated emission (ST) cross sections minus the excited state absorption (ESA) cross section (equation (4)). It has been reported that the peak of the gross gain spectrum $\gamma$ for Rh6G is red-shifted with respect to the peak of the ST cross section due to the presence of strong ESA spectrally overlapping both the GSA and ST bands, ${ }^{[51]}$ and this effect is as well observed for the sample Rh6G/pHEMA (579 nm vs. $560 \mathrm{~nm}$ ). Other wave-guiding and leaky mode effects might be affecting the shape of the gross gain spectrum $\gamma$, but there are no clear evidences of these effects.

Finally, the use of $\Delta n$ together with the losses and gross gain spectra allows obtaining the net optical gain $g$ using equation (5) at all wavelengths and pump intensities measured in the experiment, as represented in Figures 2e and $2 \mathrm{f}$. To replicate any of these graphs with the VSL method, one ought to perform 41 independent experiments (one per pump intensity). This would imply an insurmountable amount of experimental work. The fact that with the VPI method only one experiment is needed underlines the relevance and time-saving nature of this new methodology. Another great advantage of the VPI method is that it allows easily calculating the net optical gain at any pump intensity value within the experimental range using equation (2b). For the sample Rh6G/pHEMA and the pump intensity $I_{p}$ in units of $\mathrm{kW} \mathrm{cm}^{-2}$, it follows the expression:

$$
g\left(\mathrm{~cm}^{-1}\right)=20.15 \frac{\frac{I_{p}}{380.5}+\left(\frac{I_{p}}{40.6}\right)^{3.04}}{1+\frac{I_{p}}{380.5}+\left(\frac{I_{p}}{40.6}\right)^{2.8}}-14.8
$$

Note that it is not recommended to use this expression to extrapolate the net optical gain to higher pump intensity values, as the uncertainty becomes too high. 


\subsection{Comparison with gains obtained with the VSL method}

This comparison will be carried out in an asymmetric slab waveguide consisting of a 9 micron thick $10 \mathrm{mM}$ Perylene Orange (PO) PMMA film deposited onto quartz substrates (Details on experimental conditions in ref. [43]). For the VSL gains, the typical VSL equation (1) was used for the 5 weakest pump intensities, whereas for the highest ones, where gain saturation is effective, an alternative VSL expression (equation (B1)) taking into account saturation was used. ${ }^{[41]}$ For the VPI gains, the fit was performed with the GUI $A g L$ between $553 \mathrm{~nm}$ and $607 \mathrm{~nm}$ with a wavelength sampling $\Delta \lambda=1 \mathrm{~nm}$, and a stripe length $L=0.2 \mathrm{~cm}$. Only one ASE spectrum was used for the losses fit, and 22 for the full fit.

At low pump intensities, when the net gain is relatively low, both methods render similar results (Figure 3a). At higher pump intensities, on the contrary, the VPI gains are significantly smaller than the VSL ones. On first consideration, the rationale behind this behaviour could be explained claiming that the VSL method gives the "small-signal" gains and the VPI method gives the "saturated" gains corresponding to the stripe length used for the experiments. Nevertheless, whereas the latter statement is correct, the former is not entirely true.

Let us focus first on the differences between both methods. In table 1 there is a collection of the assumptions taken into account to derive equation (1) for the VSL method as compared to those to derive equations (2a) and (2b) for the VPI method. In short, the VSL expression is valid for a monochromatic steady-state photon flux propagating unidirectionally in a gain medium with a spatially homogeneous non-saturable population inversion. As explained in the introduction, this oversimplification has a profound influence on the gains obtained from the VSL method.

In any case, it is worth resorting to a qualitative description of what this oversimplification entails. For a single pump intensity, a change in the stripe length leads to a change in the real gain spatial distribution (Figure 3b). In other words, the gain depends on the stripe length $(g=g(L))$. It is easy to understand that the shorter (longer) the stripe length is, the higher (smaller) the overall gain becomes. It could be argued, and that is common belief, that this happens at long stripe lengths, 

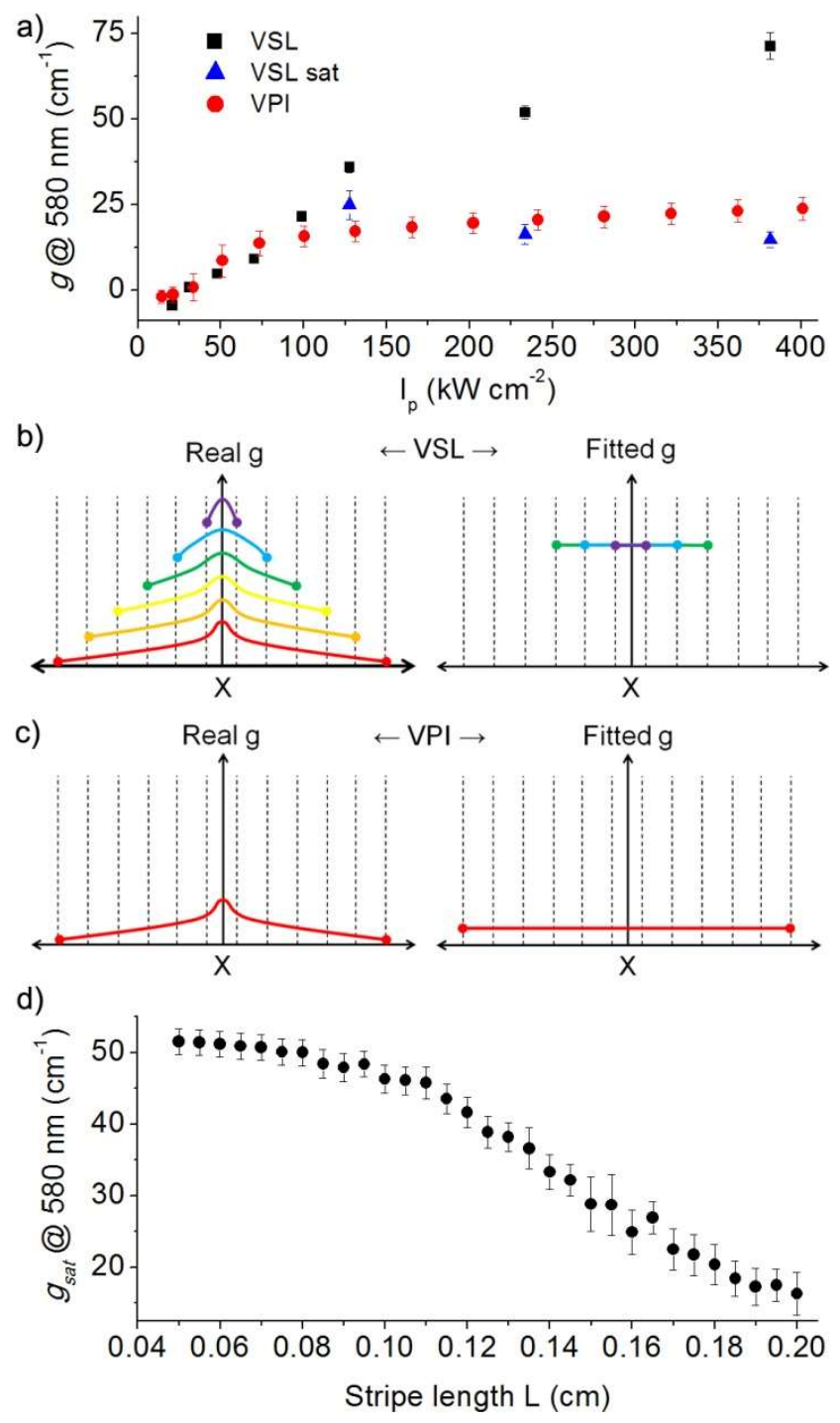

Figure 3: Gain analysis of sample PO/PMMA: a) Comparison of net optical gains obtained with the VSL method (black squares), VPI method (red dots), and assuming saturation in the VSL method (blue triangles). b) Qualitative representation of actual (left panel) and fitted (right panel) gain distributions along the excited region at a given pump value in a common VSL experiment. c) The same as b) but for the VPI experiment at the same pump value as in b). d) VSL "saturated-gain" as a function of stripe length calculated with equation (9) for a pump intensity of $230 \mathrm{~kW} \mathrm{~cm}$.

well-above ASE threshold, where there is gain saturation or depletion. But Lange et al. ${ }^{[24]}$ showed that the gain distribution is inhomogeneous even well below threshold. In contrast, the VSL equation (1) assumes that the gain is constant along the excited area and independent on the stripe length, i.e., it assigns only one gain for all stripe lengths (Figure 3b). As a consequence, the VSL method does not really give the "small-signal" gain, but a sort of spatiotemporal averaged gain of all the real gain distributions corresponding to each stripe length used for the fit (Figure 3b). Thus, the choice of the stripe length range to perform the VSL fit has a profound effect on the final gain, 
as has been recently revealed. ${ }^{[23]}$ This is one of the most critical breaches in the reliability of the VSL method and the reason why it should be used with extreme care. Anyhow, since the VSL data used for the fit are usually those for the shortest stripe lengths, the real gains will be moderately depleted and, hence, the average gain retrieved from the VSL fit will be high. On the other hand, the VPI method "suffers" a similar problem, as it assumes that the gain is constant along the stripe, but in this case assigns one gain for each pump intensity and stripe length (Figure 3c). In this sense, as the VPI method ASE spectra are usually obtained using long stripes, the real gain will be highly depleted/saturated and, consequently, the VPI method will cast a highly saturated gain value (Figure 3c). This, eventually, is the main reason why, for the same pump intensity, the VSL gain is higher than the VPI gain.

Assuming for a moment that the VSL results are reliable, it can be shown that both methods provide similar gains once saturation is taken into account in the VSL gain. In the differential equation governing the growth of ASE intensity in the VSL approximation and assuming gain saturation (equation (B2)), it can be recognized that the effective "saturated-gain" that is experienced by the flux is approximately given by:

$$
g_{s a t}=\frac{g_{s s}}{1+I / I_{s}}
$$

where $I=I(L)$ is the measured ASE intensity as a function of the stripe length, $g_{s s}$ is the small-signal gain, and $I_{s}$ is the gain saturation intensity. ${ }^{[41]}$ The last two quantities are fitting parameters obtained from the fit of equation (B1) to the plot $L$ vs. $I(L)$. The substitution into equation (9) of the $I(L)$ values and fitting parameters obtained in one of the VSL experiments shown in Figure 3a unveils, as expected, that the "saturated-gain" $g_{\text {sat }}$ decreases monotonically when increasing the stripe length (Figure 3d). At the longest stripe length, which is the same as the one used for the VPI experiment ( $L=0.2 \mathrm{~cm})$, the "saturated-gain" $g_{s a t}$ is significantly smaller than the "small-signal" gain $g_{s s}$, but it is very similar to the VPI gain (compare red dots and blue triangles in Figure 3a). In other words, both methods provide similar gains once saturation is taken into account. 
As a final comment on this analysis, the small-signal gain cannot be obtained using the VPI methodology described in the forgoing sections. To find it, one should ideally repeat the procedure of Appendix A to extend it to a bidimensional space of pump values and stripe lengths, and then proposing a new factorization for $\Delta \eta$, analogous to equations (A16)-(A17), explicitly depending on both $I_{p}$ and $L$, and probably $\lambda$. The "small-signal" gain would then be found in the limit when $L \rightarrow 0$. This task, which is beyond the scope of the present manuscript, would be highly complex, timeconsuming, and would not necessarily lead to success. In any case, it could be tackled in a future. Alternatively, to obtain a closer version of the "small-signal" gain, one could acquire the ASE spectra using the shortest possible stripe length (provided the excitation stripe is still $1 \mathrm{D}$, length $>>$ width), where saturation effects are negligible. For instance, for the sample PO/PMMA, Figure 3d suggests that stripe lengths smaller than $0.05 \mathrm{~cm}$ should be used.

\section{Concluding Remarks}

In summary, in this contribution I have found a manageable analytical expression to describe the growth of the ASE intensity as a function of the pump value (equations (2a) and (2b)) that can be fitted to experimental ASE spectra obtained in waveguides and solutions using the VPI method. This new expression is a generalization of that of the VSL method (equation (1)) and allows simultaneously retrieving, from a single VPI experiment, the losses coefficient and the net optical gains at all pump values with spectral resolution, as well as to calculate the ASE thresholds thanks to a well-defined criterion (equation (6)). The fitting procedure to retrieve all that information from the ASE spectra may not be trivially implemented. Therefore I have developed the stand-alone GUI "ASE gain Labs" $(A g L)$ to perform this task automatically. The reported methodology has been tested against ASE spectra obtained from more than 40 samples measured in different independent labs. Remarkably, the GUI $A g L$ gives reliable and consistent results and behaves strikingly well considering that none of the analysed data sets were measured with this formalism in mind. The performance against real experimental results emphasizes the relevance, power, reliability and robustness of both the VPI method and the GUI $A g L$. 
This milestone has been achieved starting from the whole set of spectrally resolved rate equations describing the spatiotemporal dynamics of ASE generation and propagation in waveguides or solutions, and following a reductionist approach based on applying a number of approximations aided by numerical simulations. Unfortunately, to find a sufficiently simple analytical solution, one must sacrifice altogether the explicit spatial variation of the population inversion and assume instead a homogeneous gain distribution. Slightly more elaborated spatial dependencies could be assumed, but the complexity of the resulting analytical solution skyrockets and the number of unknown parameters multiplies, precluding its application as a fitting expression. The "homogeneous gain distribution" assumption, which is analogous to that underlying the VSL method, together with the differences in temporal overlap between the excited state population and the photon flux at each wavelength, leads to an overestimation of the gross optical gain at the spectral edges with respect to the real ones. Fortunately, it is at the edges of the experimental spectra where the signal to noise ratio is smaller and thus the fit errors associated to it will compete with those of the overestimation. Nevertheless, both effects have to be taken into account when interpreting the results of the fit. In contrast, one of the most remarkable highlights of this manuscript is that the effective population inversion equation (2b) implicitly takes into account relevant spatiotemporal, photophysical and saturation processes, including singlet-singlet annihilation (Table 1).

At high pump intensities, well above threshold, the VPI method renders net optical gains which are significantly smaller than the VSL ones, since the latter (which is not reliable) gives the "smallsignal" gains, whereas the former retrieves the "saturated" gains corresponding to the stripe length used for the experiments. To obtain the closest version to the "small-signal" gain using the VPI method one should acquire the ASE spectra using the shortest possible stripe length (as long as it is still 1D). An important advantage of the VPI with respect to both the VSL and SES methods, is that it is immune to detection coupling artefacts leading to incorrect gain determinations, as pump and detection geometries are kept untouched, and does not suffer from inaccuracies due to gain or pump 
saturation, as these effects are implicitly taken into account in the effective population inversion (equation $(2 b)$ ).

Although the developed GUI $A g L$ is powerful enough to fit almost any kind of $I_{\text {out }}$ vs. $I_{p}$ spectra fed into it, the present methodology must only be applied to ASE spectra obtained from linear materials. That said, the formalism could be modified to find an analytical expression alternative to equation (2) to take into account non-linear effects such as Two Photon Absorption and/or some cases of reflective feedback (e.g., plane-parallel cavity [52]), albeit that would increase the formalism complexity and might even preclude its application as a fitting expression.

Finally, the results of this work are expected to have far reaching implications for the scientific community working in laser materials and devices, as it helps reducing the amount of experimental workload needed to fully characterize the ASE properties of new samples and materials (1 experiment vs. 3 experiments) and helps standardizing this kind of measurements and characterizations. In conclusion, the VPI method reported in this manuscript is a relevant, timesaver, powerful, reliable and robust formalism to retrieve simultaneously losses and optical gains from ASE spectra in active waveguides and solutions.

\section{Appendix A. Derivation of equations 2a and 2b}

\section{A.1 Photophysical processes involved and problem geometry}

The photophysical processes that have been taken into account in the present formalism are summarized in Figure (A1a). An homogeneously broadened active molecule absorbs a pump or ASE photon and is promoted from the ground (electronic) singlet manifold 0 to an excited state 3 with a probability given by the ground state absorption (GSA) cross section $\sigma_{\mathrm{GSA}}$. From this state the excited molecule relaxes down non-radiatively to a metastable electronic state 2 , and remains there until one of the following processes takes place: 
- Fluorescence emission (F): after an average time given by the fluorescence lifetime $\left(\tau_{f}\right)$, the excited molecule relaxes down spontaneously to the excited state 1 emitting a photon with a spectral distribution $F(\lambda)$ and a probability given by the quantum yield $\Phi$. From state 1 the molecule relaxes down non-radiatively to the ground state 0 .

a)

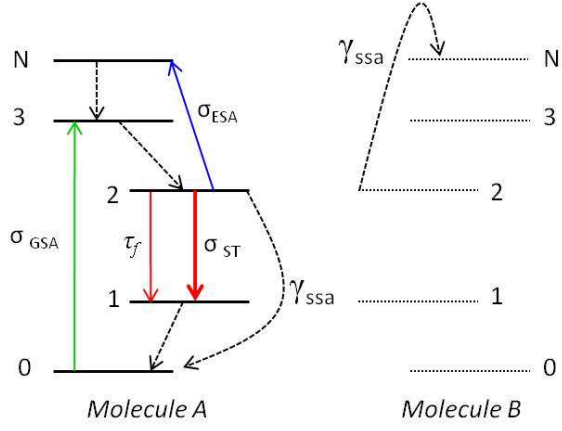

b)

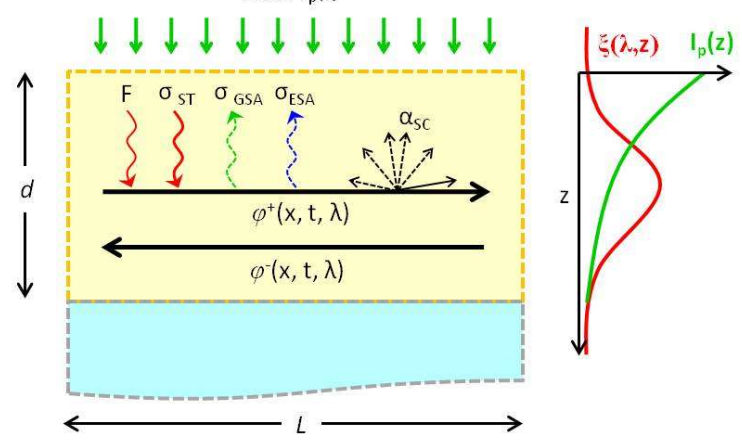

Figure A1: a) Jablonsky diagram showing the photophysical processes considered in the present formalism. The solid and dashed arrows represent, respectively, radiative and non-radiative transitions. b) Sketch of the waveguide geometry and processes taken into account in the methodology. For a description of the notation see the text.

- Stimulated emission (ST): the excited molecule is "forced" to relax down to the electronic state 1 emitting a photon identical to the stimulating one with a probability given by the stimulated emission cross section $\sigma_{\mathrm{ST}}$. From state 1 the molecule relaxes down non-radiatively to the ground state 0.

- Excited state absorption (ESA): the excited molecule absorbs an ASE photon and is promoted to an upper excited state $\mathrm{N}$ with a probability given by the excited state absorption cross section $\sigma_{\mathrm{ESA}}$. From state $\mathrm{N}$ the molecule relaxes down non-radiatively to the excited state 2 again. 
- Singlet-singlet annihilation (SSA): the excited molecule $A$ encounters another excited molecule $B$ and, mediated by a Förster type non-radiative energy transfer with strength proportional to $\gamma_{\text {ssa }}, A$ is relaxed down non-radiatively directly to the ground state 0 and $B$ is promoted to an upper excited state N. From state N the molecule relaxes down non-radiatively to the excited state 2 again.

The geometry of the system will consist of a transversally pumped excited region of length $L$, width $b(L>>b)$ and depth $d$, determined by the pump focusing and film thickness (or pump penetration depth if dealing with solutions), respectively. Figure A1b shows a scheme with the different processes taken into account in the simulation. Due to the pumping geometry, the ASE grows preferentially in the direction defined by the axis of the excited region, which is perpendicular to the pump direction. Two counter propagating modes along the axis of the excited region are considered: the forward $\left(\varphi^{+}(\mathrm{x}, \mathrm{t}, \lambda)\right)$ and backward $\left(\varphi^{-}(\mathrm{x}, \mathrm{t}, \lambda)\right)$ photon fluxes. The fluxes $\varphi^{ \pm}(\mathrm{x}, \mathrm{t}, \lambda)$ are seeded by spontaneous emission $(\mathrm{F})$, amplified by stimulated emission $\left(\sigma_{\mathrm{ST}}\right)$, reabsorbed by both the ground and excited state populations $\left(\sigma_{\mathrm{GSA}}\right.$ and $\left.\sigma_{\mathrm{ESA}}\right)$ and dispersed due to scattering $\left(\alpha_{\mathrm{SC}}\right)$.

\section{A.2 System of equations and numerical solutions}

To model the emission processes involved in transversally pumped active media the rate equations formalism has been used. The formalism and notation will be similar to those employed previously, ${ }^{[28]}$ but with some added terms to take into account singlet-singlet annihilation ${ }^{[53,54]}$ and waveguide mode propagation effects. ${ }^{[29-32]}$

Assuming that the pump intensity $\left(I_{p}\right)$ is kept within realistic limits, the populations of states 3 and 1 can be eliminated adiabatically, i.e., $\partial N_{3} / \partial \mathrm{t}=\partial N_{l} / \partial \mathrm{t}=N_{3}=N_{1}=0$. Consequently, the population in state 2 will effectively account for the population inversion (i.e., $\Delta N=N_{2}-N_{1} \approx N_{2}$ ). With these assumptions, the modified set of rate equations reads: 


$$
\begin{array}{r}
\frac{\partial N_{2}(x, t)}{\partial t}=\sigma_{G S A}(\lambda p) \frac{I_{p}(t)}{h v_{p}} N_{0}(x, t)-\frac{N_{2}(x, t)}{\tau_{f}}-\gamma_{s s a} N_{2}(x, t)^{2} \\
-N_{2}(x, t) \int \Gamma(\lambda) \sigma_{S T}(\lambda)\left(\varphi^{+}(x, t, \lambda)+\varphi^{-}(x, t, \lambda)\right) d \lambda \\
+N_{0}(x, t) \int \Gamma(\lambda) \sigma_{G S A}(\lambda)\left(\varphi^{+}(x, t, \lambda)+\varphi^{-}(x, t, \lambda)\right) d \lambda \\
N_{0}=n_{d}-N_{2} \\
\left(\frac{n_{r}}{c} \frac{\partial}{\partial t} \pm \frac{\partial}{\partial x}\right) \varphi^{ \pm}(x, t, \lambda)=\beta \Phi \frac{N_{2}(x, t)}{\tau_{f}} F(\lambda) \\
+\Gamma(\lambda) \sigma_{S T}(\lambda) N_{2}(x, t) \varphi^{ \pm}(x, t, \lambda) \\
-\Gamma(\lambda) \sigma_{G S A}(\lambda) N_{0}(x, t) \varphi^{ \pm}(x, t, \lambda) \\
-\Gamma(\lambda) \sigma_{E S A}(\lambda) N_{2}(x, t) \varphi^{ \pm}(x, t, \lambda) \\
-\alpha \varphi_{S C}(\lambda) \varphi^{ \pm}(x, t, \lambda)
\end{array}
$$

$N_{0}(x, t)$ and $N_{2}(x, t)$ are the population densities $\left(\right.$ molecules $\left.\times \mathrm{cm}^{-3}\right)$ in the ground and excited states, respectively. The mode confinement function $\Gamma(\lambda)$ garners all information on the overlap between the pump intensity variation $I_{p}(\mathrm{z})$ across the film and the mode profile $\xi(\lambda, \mathrm{z})$ (Figure Alb and refs [29-31]), as well as information on all possible propagation and interference effects (e.g., leakymodes [32]). $n_{d}$ is the volume concentration of active centres. The $\beta$ factor contains information on the percentage of light that is coupled into the waveguide modes. $F(\lambda)$ is the fluorescence spectrum (as measured in a spectrofluorimeter) normalized to unity. Finally, $v_{p}, n_{r}, h$ and $c$ are the pump photon frequency, the refractive index of the film, the Planck's constant, and the speed of light in vacuum, respectively.

The right-hand terms in eq. (A1) describe, from left to right: absorption of the pumping flux, spontaneous decay through all available channels, singlet-singlet annihilation, ${ }^{[53,54]}$ depletion of the excited state due to stimulated emission of radiation in all wavelengths, and recovery of population due to self-absorption of the photon flux. The right-hand terms in eq. (A3) are interpreted as follows: The spontaneous emission coupled to the waveguide mode (first term) is amplified by stimulated emission (second term), reabsorbed by the ground (third term) and excited (fourth term) 
states populations, and scrambled due to scattering and modal losses (last term). For the sake of notation simplicity, it is convenient to perform the following change of variables:

$$
\begin{gathered}
n_{i}(x, t)=\frac{N_{i}(x, t)}{n_{d}} ; i=0,2 \\
\tilde{t}=\frac{t}{\tau_{f}} \\
\phi(x, \tilde{t}, \lambda)=\varphi(x, t, \lambda) \frac{\tau_{f}}{n_{d}} \\
W_{i}(x, \tilde{t})=\int \alpha_{i}(\lambda) \Gamma(\lambda)\left(\phi^{+}(x, \tilde{t}, \lambda)+\phi^{-}(x, \tilde{t}, \lambda)\right) d \lambda ; i=S T, G S A, E S A \\
\gamma_{S S}=\gamma_{S S a} n_{d} \tau_{f} \\
I_{p s}=\frac{h v_{p}}{\sigma_{G S A}\left(\lambda_{p}\right) \tau_{f}} \\
\Omega(\lambda)=\beta \Phi F(\lambda)=\Omega_{0} F(\lambda) \\
\alpha=\alpha_{i}(\lambda)=\Gamma(\lambda)\left(\alpha_{S C}(\lambda)+\alpha_{G S A}(\lambda)\right) \\
\tilde{\gamma}=\tilde{\gamma}(\lambda)=\Gamma(\lambda)\left(\alpha_{S T}(\lambda)+\alpha T_{G S A}(\lambda)-\alpha_{E S A}(\lambda)\right),
\end{gathered}
$$

where the last two parameters $\alpha$ and $\tilde{\gamma}$ account for the total losses and the gross optical gain coefficients, respectively. Note that the normalized populations $n_{i}$ are now adimensional (Eq. (A4a)). Substituting eq. (A2) into eqs. (A1) and (A3), and applying into the resulting equations the normalizations and change of variables (A4) results into the simplified set of equations:

$$
\begin{aligned}
& \frac{\partial n_{2}(x, \tilde{t})}{\partial \widetilde{t}}=\frac{I_{p}(\tilde{t})}{I_{p s}}+W_{G S A}(x, \tilde{t})-\gamma_{S S} n_{2}(x, \widetilde{t})^{2} \\
& \quad-n_{2}(x, \tilde{t})\left(1+\frac{I_{p}(\tilde{t})}{I_{p s}}+W_{S T}(x, \tilde{t})+W_{G S A}(x, \tilde{t})\right)
\end{aligned}
$$




$$
\begin{aligned}
&\left(\frac{n_{r}}{c \tau_{f}} \frac{\partial}{\partial \widetilde{t}} \pm \frac{\partial}{\partial x}\right) \phi^{ \pm}(x, \widetilde{t}, \lambda)=\Omega(\lambda) n_{2}(x, \tilde{t}) \\
&+\left(\widetilde{\gamma} n_{2}(x, \widetilde{t})-\alpha\right) \phi^{ \pm}(x, \tilde{t}, \lambda)
\end{aligned}
$$

This system of equations was solved numerically to recreate a customary experimental configuration: a polymer film $\left(n_{r}=1.49\right)$ of thickness $d=1 \mu \mathrm{m}$ and doped with a generic homogeneously broadened dye $\left(\tau_{f}=6 \mathrm{~ns} ; \Phi=0.8 ; n_{d}=6 \times 10^{18} \mathrm{~cm}^{-3}(10 \mathrm{mM}) ; \gamma_{s s a}=2 \times 10^{11} \mathrm{~cm}^{3} \mathrm{~s}^{-1}\right)$ is transversally pumped at a wavelength of $532 \mathrm{~nm}$ by a laser beam with a Gaussian temporal shape, spatially conformed as a stripe of length $L=2 \mathrm{~mm}$ and width $b=150 \mu \mathrm{m}$. The spectral extinction coefficients $\alpha_{i}$ (with $i=$ GSA, ST, and ESA) and the gross gain coefficient $\tilde{\gamma}$ corresponding to this generic dye are displayed in figure A2a. The problem was solved for several pump energies with
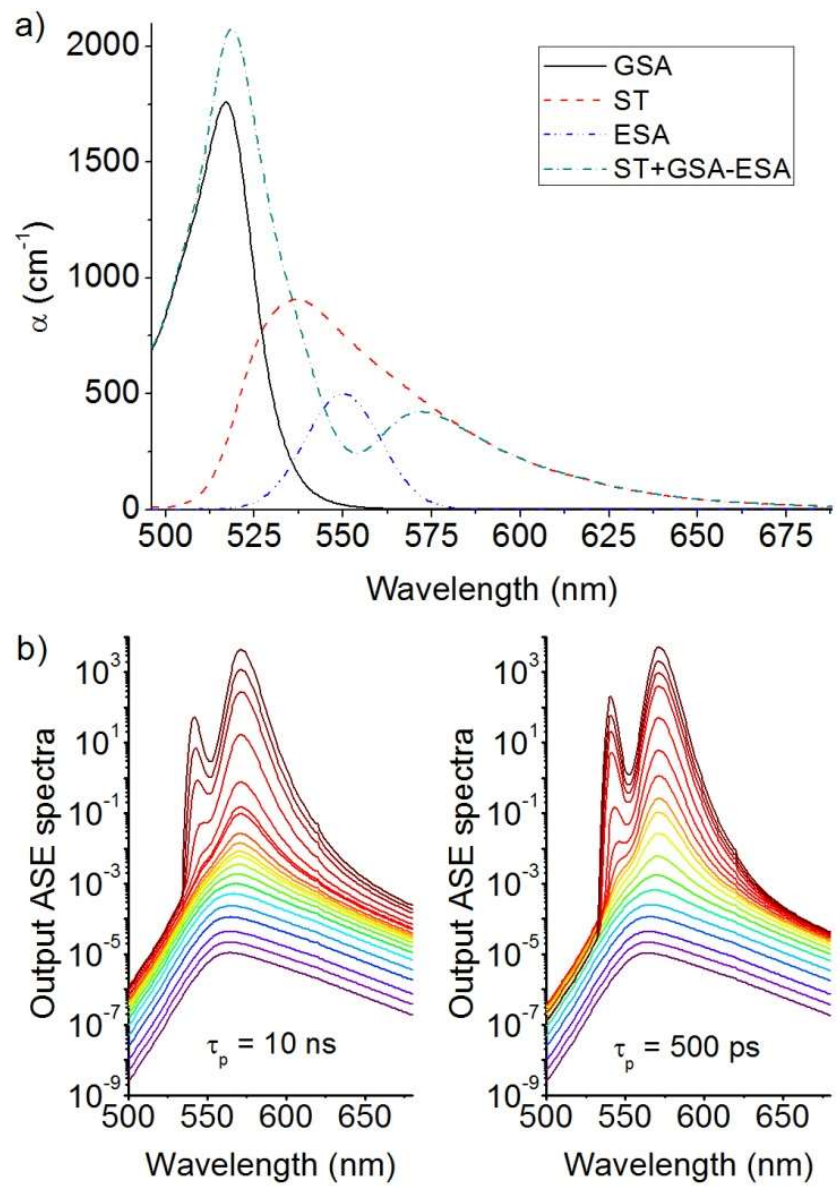

Figure A2: a) Spectral extinction and gain coefficients $\alpha_{i}$ (with $i=$ GSA, ST, and ESA) corresponding to the generic dye used in the simulations. b) Simulated temporally integrated output ASE spectra $I_{A S E}\left(L, I_{p}, \lambda\right)$ for pump pulse durations of $\tau_{\mathrm{p}}=10 \mathrm{~ns}$ (left) and $\tau_{\mathrm{p}}=500 \mathrm{ps}$ (right). 
two different pump pulse durations: $\tau_{\mathrm{p}}=10 \mathrm{~ns}\left(>\tau_{\mathrm{f}}\right)$ and $\tau_{\mathrm{p}}=500 \mathrm{ps}\left(<\tau_{\mathrm{f}}\right)$. The temporally integrated output ASE spectra $I_{A S E}(L, \lambda)=\int \phi(x=L, \lambda, \tilde{t}) d \widetilde{t}$, which corresponds to what one would measure in a lab, are displayed in figure A2b.

\section{A.3 Analytical solution (Generalization of VSL equation)}

The goal of this formalism is to find an analytical expression from the system of eqs. (A5)-(A6) that can be fitted to the experimental ASE spectra. By all means, there is no way of exactly solving this system of coupled non-linear equations and thus a number of assumptions must be done, and adequate approximations must be applied. To start with, the following Heuristic ansatz is tried. The re-normalized photon fluxes $\square^{ \pm}$(focusing from now on in $\square^{+}$and dropping the ${ }^{+}$subscript for the sake of notation simplicity) are factorized as:

$$
\phi(x, \lambda, \tilde{t}):=I(x, \lambda) \tau_{i}(\lambda, \tilde{t})
$$

With:

$$
\begin{gathered}
I(x, \lambda)=\int_{-\infty}^{\infty} \phi(x, \lambda, \tilde{t}) d \tilde{t} \\
\tau_{i}(\lambda, \tilde{t})=\frac{\int_{0}^{L} \phi(x, \lambda, \tilde{t}) d x}{\int_{0}^{L} d x \int_{-\infty}^{\infty} \phi(x, \lambda, \tilde{t}) d \tilde{t}}
\end{gathered}
$$

The temporally integrated ASE intensity $I(x, \lambda)$ and the function $\tau_{i}$ gather the information about the spatial and temporal behaviour of the photon flux $\square$, respectively. Note that $\tau_{i}$ is normalized so that:

$$
\begin{aligned}
& \int_{-\infty}^{\infty} \tau_{i}(\lambda, \widetilde{t}) d \widetilde{t}=1 \\
& \lim _{\widetilde{t} \rightarrow \pm \infty} \tau_{i}(\lambda, \widetilde{t})=0
\end{aligned}
$$


where the asymptotes in eq. (A10b) originate from the "pulse-like" temporal dependency of $\square$. Substituting the factorization (A7) into eq. (A6) (the excited state population eq. (A5) is not needed anymore for the current method), we arrive to:

$$
\frac{n_{r}}{c \tau_{f}} I \frac{d \tau_{i}}{d \widetilde{t}}+\tau_{i} \frac{d I}{d x}=\Omega n_{2}+\left(\widetilde{\gamma} n_{2}-\alpha\right) I \tau_{i}
$$

For notation simplicity, the implicit and explicit dependencies have been set aside temporarily. Multiplying both sides of eq. (A11) by $d \tilde{t}$, integrating between $\pm \infty$, and applying the conditions (A10), we find that:

$$
\frac{d I}{d x}=\Omega \eta+(\tilde{\gamma} \Delta \eta-\alpha) I
$$

with

$$
\begin{gathered}
\eta=\eta(x)=\int_{-\infty}^{\infty} n_{2}(x, \tilde{t}) d \tilde{t} \\
\Delta \eta=\Delta \eta(x, \lambda)=\int_{-\infty}^{\infty} n_{2}(x, \tilde{t}) \tau_{i}(\lambda, \tilde{t}) d \tilde{t}
\end{gathered}
$$

Although the functions $\eta$ and $\Delta \eta$ represent different measures of the temporally integrated population inversion, the latter quantifies how well the population inversion $n_{2}$ and the photon flux $\square$, or more precisely $\tau_{i}$, overlap in time. Note that $\Delta \eta$ displays a spectral dependence, as each wavelength of the photon flux has its particular temporal behaviour and, consequently, will experience a different population inversion. Obviously, under $\mathrm{CW}$ excitation $\Delta \eta$ would lose this spectral dependency and would equal $\eta$.

The general solution for the linear first-order ordinary differential eq. (A12) as obtained using the integrating factor method is given by:

$$
I_{A S E}(\lambda, L)=\left.\Omega e^{\int\left(\tilde{\gamma} \Delta \eta\left(x^{\prime}\right)-\alpha\right) d x^{\prime}}\right|_{x^{\prime}=L} \int_{0}^{L}\left(\eta(x) e^{-\left.\int\left(\widetilde{\gamma} \Delta \eta\left(x^{\prime}\right)-\alpha\right) d x^{\prime}\right|_{x^{\prime}=x}}\right) d x
$$


The particular solution will eventually depend on the spatial dependency of $\eta$ and $\Delta \eta$. These functions have a bell-shape spatial dependency ${ }^{[24,28]}$ that leads to a solution with indefinite integrals which cannot be solved in general (neither analytically nor numerically). Unfortunately, to find a sufficiently simple analytical solution for eq. (A14), one must sacrifice altogether the explicit spatial variation of the population inversions $\eta(x)$ and $\Delta \eta(x)$ and use spatial averages instead (i.e, homogeneous gain distribution). Slightly more elaborated spatial dependencies could be assumed, but the complexity of the resulting analytical solution skyrockets and the number of unknown parameters multiplies, precluding its application as a fitting expression. Thus, the particular solution for eq. (A14) assuming a spatially homogeneous population inversion (i.e, $\eta(x):=\bar{\eta}$ and $\Delta \eta(x):=\overline{\Delta \eta})$ reads:

$$
I_{A S E}\left(\lambda, I_{p}, L\right)=\frac{\Omega(\lambda) \bar{\eta}\left(I_{p}, L\right)}{\tilde{\gamma}(\lambda) \overline{\Delta \eta}\left(\lambda, I_{p}, L\right)-\alpha(\lambda)}\left(e^{\left(\tilde{\gamma}(\lambda) \overline{\Delta \eta}\left(\lambda, I_{p}, L\right)-\alpha(\lambda)\right) L}-1\right)
$$

It is easy to see that this equation is a generalization of the common VSL expression eq. (1) that has been used for decades. In eq. (A15) all the explicit and implicit dependencies have been recovered. It is important to note that, unlike $I(x, \lambda)$ or $\tau_{i}$, the newly assumed spatial averages $\bar{\eta}$ and $\overline{\Delta \eta}$ have no clear explicit expression within this approximation due to the complex non-linear coupled nature of the system of eqs. (A5) and (A6). Nevertheless, they depend implicitly on the stripe length $(L)$, pump intensity $\left(I_{p}\right)$ and wavelength in a complex fashion that will transpire later on. That said, as the main objective of this work is to retrieve the optical gains from VPI experiments, the implicit dependency on $I_{p}$ will be left through the rest of the manuscript, whereas the stripe length one will be dropped for the sake of notation simplicity. 


\section{A.4 A further simplification for $\overline{\Delta \eta}$}

To further proceed, let's focus on the temporally integrated spatially averaged (TISA) population inversion $\overline{\Delta \eta}$. Although Eq. (A15) could be used for both the VSL and VPI methods, we want to apply it to the latter. Hence, the question arises: how does $\overline{\Delta \eta}$ depend on the pump intensity? As I have just mentioned, there is no explicit expression for the function $\overline{\Delta \eta}$ and, accordingly, it must be retrieved by an indirect method. It consists of fitting eq. (A15) to the output ASE spectra obtained numerically in section A.2 (figure A2b). Since $\tilde{\gamma}(\lambda), \alpha(\lambda), \Omega(\lambda)$, and $L$ are known exactly, the only unknowns are $\overline{\Delta \eta}\left(\lambda, I_{p}\right)$ and $\bar{\eta}\left(I_{p}\right)$, which are left free.

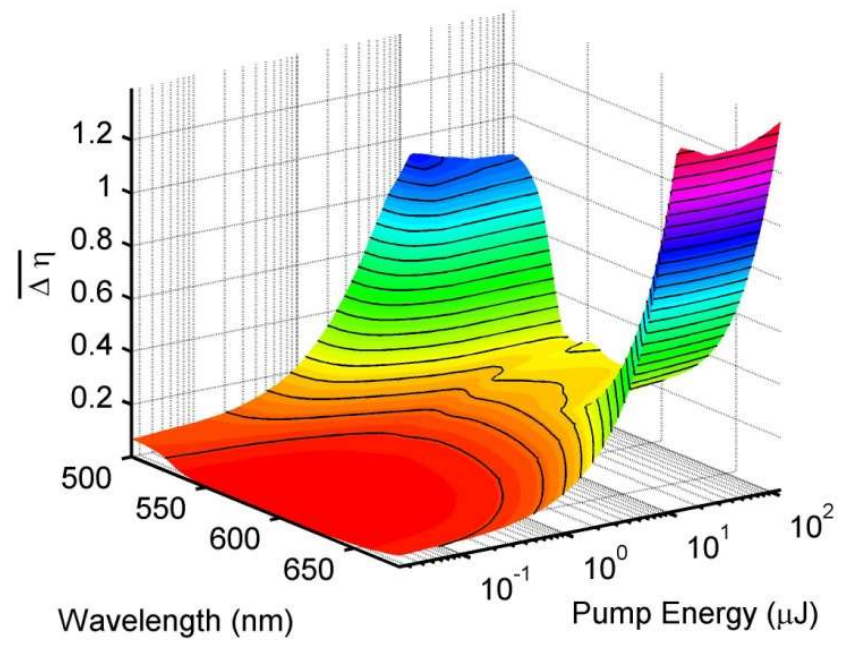

Figure A3: Temporally integrated spatially averaged population inversion $\overline{\Delta \eta}$ experienced by the photon flux $\square\left(\tau_{\mathrm{p}}=10 \mathrm{~ns}\right)$.

The computed $\overline{\Delta \eta}$ has a strong dependency on both the emitted wavelength and the pump value (Figure A3). In this sense, a change in the pump pulse duration results in a distortion of its shape, but not of its general trends. As expected, the population inversion builds up globally with the pump energy following a characteristic S-shape function displaying saturation behaviour. ${ }^{[5]}$ Perhaps more striking is that the fitted TISA population inversion experienced at the spectral edges (regions of low gain or high losses) is significantly stronger than that experienced at the region of maximal gain $(\sim 570 \mathrm{~nm})$. One would expect, on the contrary, a better temporal overlap (cf. eq. (A13b)) of the 
actual population inversion $n_{2}$ and the photon flux at the wavelengths of high gain, and in fact this is the case (result not shown). Unfortunately, this counter-intuitive behaviour is a side effect stemming from assuming that both $\eta(x)$ and $\Delta \eta(x)$ are spatially homogenous, and is the price to pay for the simplified analytical expression eq. (A15).

Given the dependency of $\overline{\Delta \eta}$ on both the emitted wavelength and the pump intensity, it is not possible to fit eq. (A15) as it is to the experimental data, as there would be more variables (unknowns) than experimental points (equations), and the system would be underdetermined. In other words, it would have an infinite number of solutions. Thus, the system must be given constraints and that implies doing a further approximation. It consists of factorizing ad hoc the TISA population inversion $\overline{\Delta \eta}$ as:

$$
\overline{\Delta \eta}\left(\lambda, I_{p}\right):=\xi(\lambda) \Delta n\left(I_{p}\right)
$$

The "spectral correction" function $\xi(\lambda)$, which is normalized so that $\int \xi(\lambda) d \lambda=1$, takes into account the differences in amplification strength as a function of wavelength and, implicitly, the stripe length. On the other hand, the effective population inversion $\Delta n\left(I_{p}\right)$ contains information on the dependency on both the pump intensity and the stripe length. $\xi(\lambda)$ and $\Delta n\left(I_{p}\right)$ can be retrieved in a similar fashion as was done for $\overline{\Delta \eta}\left(\lambda, I_{p}\right)$. In this case, eq. (A15), together with the factorization (A16), is fitted to the values of $I_{A S E}\left(\lambda, I_{p}, L\right)$ obtained numerically (figure A2b), with $\xi(\lambda)$ and $\Delta n\left(I_{p}\right)$ acting as free fitting parameters. The results of this procedure can be found in Figure A4.

With a view to avoiding uncertainties related to the fitting procedure and easing the post-fitting data manipulation, it is fundamental to find a formula to express $\Delta n\left(I_{p}\right)$ as an explicit function of $I_{p}$. It would be tempting to use the expression for the population inversion in a four-level laser system typically found in laser physics textbooks, ${ }^{[55]}$ i.e., $\Delta n\left(I_{p}\right) \propto\left(I_{p} / I^{s} p\right) /\left(1+\left(I_{p} / I_{p}^{s}\right)\right)$. Unfortunately, such simplified expression is not sufficiently accurate and cannot capture the subtle details needed to perform a good fit. Following a heuristic method, I have found that $\Delta n\left(I_{p}\right)$ can be described 

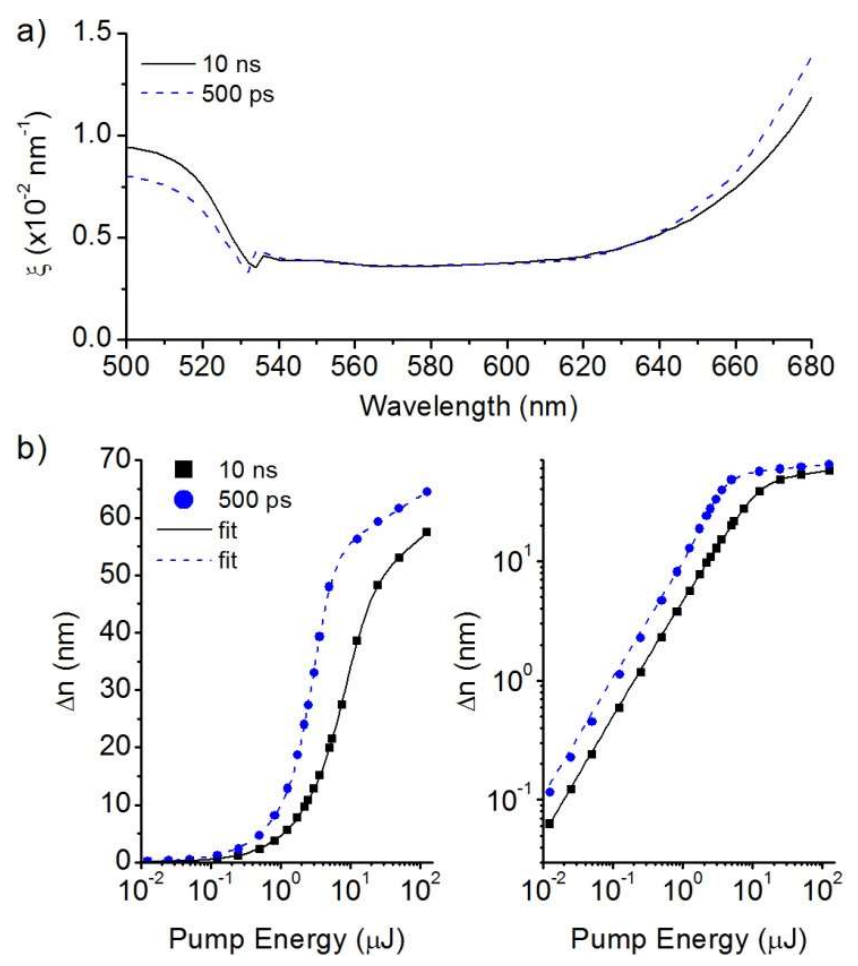

Figure A4: a) "Spectral correction" function as a function of emission wavelength for two pump pulse durations. b) Effective population inversion as a function of pump energy represented in semi-log x scale (left) and log-log scale (right). In b) the dots represent the values obtained numerically and the lines show the best fit of eq. ( $2 b)$ to the numerical values.

exceptionally well, both at low and high pump intensities (Figure A4b), with the more elaborated expression of eq. (2b).

Finally, the substitution of the ansatz (A16) for the population inversion into eq. (A15) leads to eq. (2a). Note that the bar over the spatial average $\bar{\eta}$ has been removed for notation simplicity.

\section{Appendix B. Equations for the VSL method taking gain saturation into account}

The growth of ASE intensity $(I(L))$ as a function of stripe length $L$ in the presence of gain saturation is given by: ${ }^{[41]}$

$$
L=\frac{I(L) / I_{s}}{g_{s s}+\Omega / I_{s}}+\frac{g_{s s}}{\left(g_{s s}+\Omega / I_{s}\right)^{2}} \ln \left|\frac{\Omega+\left(g_{s s}+\Omega / I_{s}\right) I(L)}{\Omega}\right|
$$

Expression which is obtained integrating the following differential equation:

$$
\frac{d I}{d x} \approx \Omega+\frac{g_{S S} I}{1+I / I_{S}}
$$


Eq. (B1) is implicit in $I(L)$, and thus, the gain $g_{s s}$ is obtained from a fit of eq. (B1) to the plot $L$ vs. $I(L) \cdot{ }^{[41]}$

\section{Appendix C. Supplementary Material}

It contains supplementary text and figures: Section S1, The complete fitting procedure; Section S2, Performance and sensitivity to noise of VPI method and GUI $A g L$.

\section{Acknowledgements}

The author thanks Dr. K. Kazlauskas (Vilnius Univ., Lithuania), Dr. M. Anni (Univ. of Salento, Italy), and Dr. M. A. Díaz-García (Univ. de Alicante, Spain), for kindly lending their ASE spectra obtained from their samples to perform independent tests to the new methodology. In addition, he acknowledges N. Fonseca-Bonilla, MSc (CAB INTA-CSIC, Spain) for contributing with fruitful discussions and for appraising and proof-reading the manuscript.

\section{References}

[1] A. J. C. Kuehne and M. C. Gather, Chem. Rev. 116, 12823-12864 (2016).

[2] L. Cerdán, State-of-the-art active materials for organic lasers, in: M. Anni and S. Lattante (eds.), Organic Lasers: Fundamentals, Developments, and Applications (Pan Stanford Publishing, Singapore, 2018), chap. 3.

[3] L. Cerdán, J. Braborec, A. Costela, I. García-Moreno, and M. G. S. Londesborough, Nat. Commun. 6, 5958 (2015).

[4] F. Fan, O. Voznyy, R. P. Sabatini, K.T. Bicanic, M.M. Adachi, J.. McBride, K.R. Reid, Y.-S. Park, X. Li, A. Jain, R. Quintero-Bermudez, M. Saravanapavanantham, M. Lui, M. Korkusinski, P. Hawrylak, V.I. Klimov, S.J. Rosenthal, S. Hoogland, and E.H. Sargent, Nature 544, 75-79 (2017).

[5] B. Guzelturk, M. Pelton, M. Olutas, and H. V. Demir, Nano Lett. 19, 277-282 (2019).

[6] C. Grivas and M. Pollnau, Laser Photonics Rev. 6, 419-462 (2012). 
[7] P. Brenner, T. Glöckler, D. Rueda-Delgado, T. Abzieher, M. Jakoby, B. S. Richards, U. W. Paetzold, I. A. Howard, and U. Lemmer, Opt. Mater. Express 7, 4082-4094 (2017).

[8] J. R. Harwell, G.L. Whitworth, G.A. Turnbull, I.D.W. Samuel, Sci. Rep. 7, 11727 (2017).

[9] C. Grivas, Prog. Quantum Electron. 35, 159-239 (2011).

[10] C. Grivas, Prog. Quantum Electron. 45-46, 3-160 (2016).

[11] M. Anni and S. Lattante (eds.), Organic Lasers: Fundamentals, Developments, and Applications (Pan Stanford Publishing, Singapore, 2018).

[12] A.L. Dobryakov, S.A. Kovalenko, A. Weigel, J.L.Pérez-Lustres, J. Lange, A. Müller, and N.P. Enrsting, Rev. Sci. Instrum. 81, 113106 (2010).

[13] P.C. Mogensen, P.M. Smowton, and P. Blood, Appl. Phys. Lett. 71, 1975 (1997).

[14] J. Valenta, I. Pelant, and J. Linnros, Appl. Phys. Lett. 81, 1396-1398 (2002).

[15] K. L. Shaklee and R. F. Leheny, Appl. Phys. Lett. 18, 475-477 (1971).

[16] Y. Sorek, R. Reisfeld, I. Finkelstein, and S. Ruschin, Appl. Phys. Lett. 66, 1169-1171 (1995).

[17] M. Vehse, J. Mainertz, O. Lange, P. Michler, J. Gutowski, S. Bader, A. Lell, and V. Härle, Phys. Stat. Sol. (c) 0, 43-47 (2002).

[18] C. Lange, M. Schwalm, S. Chatterjee, W. W. Rühle, N. C. Gerhardt, S. R. Johnson, J.-B. Wang, and Y.-H. Zhang, Appl. Phys. Lett. 91, 191107 (2007).

[19] A. Costela, O. García, L. Cerdán, I. García-Moreno, and R. Sastre, Opt. Express 16, 7023$7036(2008)$.

[20] A. Hariri and S. Sarikhani, J. Mod. Opt. 62, 822-829 (2015).

[21] F. Vogelbacher, X. Zhou, J. Huang, M. Li, K.-J. Jiang, Y. Song, K. Unterrainer, and R. Hainberger, Opt. Mater. Express 9, 1208-1222 (2019)

[22] R. Muñoz-Mármol, N. Zink-Lorre, J. M. Villalvilla, P. G. Boj, J. A. Quintana, C. Vázquez, A. Anderson, M. J. Gordon, A. Sastre-Santos, F. Fernández-Lázaro, and M. A. Díaz-García, J. Phys. Chem. C 122, 24896-24906 (2018).

[23] L. Cerdán, Opt. Lett. 42, 5258-5261 (2017). 
[24] C. Lange, M. Schwalm, B. Metzger, and S. Chatterjee, J. Appl. Phys. 108, 103119 (2010).

[25] I. Silvestre, P. W. B. Marques, M. Valaderes, and L. A. Cury, Appl. Phys. Lett. 93, 163307 (2008).

[26] S. Chénais and S. Forget, Appl. Phys. Lett. 94, 106101 (2009).

[27] I. Silvestre, P. W. B. Marques, M. Valaderes, and L. A. Cury, Appl. Phys. Lett. 94, 106102 (2009).

[28] L. Cerdán, A. Costela, I. García-Moreno, V. Martín and M. E. Pérez-Ojeda, IEEE J. Quantum Electron. 47, 907-919 (2011).

[29] M. Anni, A. Perulli, and G. Monti, J. Appl. Phys. 111, 093109 (2012).

[30] J. Arrue, M. A. Illarramendi, I. Ayesta, F. Jimémez, J. Zubia, A. Tagaya, and Y. Koike, IEEE Photonics J. 7, 7100611 (2015).

[31] I. Gozhyk, M. Boudreau, H. Rabbani Haghighi, N. Djellali, S. Forget, S. Chénais, C. Ulysse, A. Brosseau, R. Pansu, J.-F. Audibert, S. Gauvin, J. Zyss, and M. Lebental, Phys. Rev. B 92, $214202(2015)$.

[32] A. Penzkofer, W. Holzer, H. Tillmann, H.-H. Hörhold, Opt. Commun. 229, 279-290 (2004).

[33] O. Svelto, Principles of Lasers (Springer, 1998).

[34] L. Dal Negro, P. Bettotti, M. Cazzanelli, D. Pacifici, and L. Pavesi, Opt. Commun. 229, 337-348 (2004).

[35] K. Kazlauskas, G. Kreiza, O. Bobrovas, O. Adomeniene, P. Adomenas, V. Jankauskas, and S. Jursenas, Appl. Phys. Lett. 107, 0433301 (2015).

[36] H. Azuma, T. Kobayashi, Y. Shim, N. Mamedov, H. Naito, Org. Electron. 8, 184-188 (2007)

[37] M. Anni and S. Lattante, J. Phys. Chem. C 119, 21620-21625 (2015).

[38] Z. E. Lampert, J. M. Papanikolas, S. E. Lappi, and C. Lewis Reynolds Jr. Opt. Laser Technol. 94, 77-85 (2017). 
[39] F. Lahoz, C. J. Oton, N. Capuj, M. Ferrer-González, S. Cheylan, and D. Navarro-Urrios, Opt. Express 17, 16766-16775 (2009).

[40] L. Cerdán, A. Costela, I. García-Moreno, O. García, and R. Sastre, Appl. Phys. B 97, 73-83 (2009).

[41] L. Cerdán, A. Costela, and I. García-Moreno, J. Opt. Soc. Am. B 27, 1874-1877 (2010).

[42] L. Cerdán, A. Costela, I. García-Moreno, O. García, R. Sastre, M. Calle, D. Muñoz and J. de Abajo, Macromol. Chem. Phys. 210, 1624-1631 (2009).

[43] L. Cerdán, A. Costela, G. Durán-Sampedro and I. García-Moreno, M. Calle, M. Juan-y-Seva, J. de Abajo, and G. A. Turnbull, J. Mater. Chem. 22, 8938-8947 (2012).

[44] L. Cerdán, A. Costela, and I. García-Moreno, Org. Electron. 13, 1463-1469 (2012).

[45] L. Cerdán, A. Costela, G. Durán-Sampedro and I. García-Moreno, Appl. Phys. B 108, 839850 (2012).

[46] L. Cerdán, V. Martínez-Martínez, I. García-Moreno, A. Costela, M. E. Pérez-Ojeda, I. López Arbeloa, L. Wu, and K. Burgess, Adv. Opt. Mater. 1, 984-990 (2013).

[47] M. Alcaire, L. Cerdán, F. Lahoz, F. J. Aparicio, J. C. González, F. J. Ferrer, A. Borrás, J. P. Espinós, and A. Barranco, ACS Appl. Mater. Interfaces. 9, 8948-8959 (2017).

[48] M. Morales-Vidal, P. G. Boj, J. M. Villalvilla, J. A. Quintana, Q. Yan, N.-T. Lin, X. Zhu, N. Ruangsupapichat, J. Casado, H. Tsuji, E. Nakamura, and M. A. Díaz-García, Nat. Comm. 6, 8458 (2015).

[49] K. Kazlauskas, G. Kreiza, E. Radiunas, P. Adomenas, O. Adomeniene, K. Karpavicius, J. Bucevicius, V. Jankauskas, and S. Jursenas, Phys. Chem. Chem. Phys. 17, 12935 (2015).

[50] H. Goudket, T.-H. Nhung, B. Ea-Kim, G. Roger, and M. Canva, Appl. Opt. 45, 7736-7741 (2006).

[51] P. R. Hammond, IEEE J. Quantum Electron. QE-15, 624-632 (1979).

[52] R. Weigand and J. M. Guerra, Opt. Quant. Electron. 27, 1027-1051 (1995). 
[53] S. Y. Arzhantsev, A. Y. Chikishev, N. I. Koroteev, S. A. Magnitskii, and V. V. Shubin, Laser Physics 9, 466-469 (1999).

[54] S. M. King, D. Dai, C. Rothe, and A. P. Monkman, Phys. Rev. B 76, 085204 (2007).

[55] A. E. Siegmann, Lasers (University Science Books, 1986) 


\title{
Supplementary Material
}

\author{
for \\ Simultaneous retrieval of optical gains, losses, and threshold in active \\ waveguides \\ Luis Cerdán ${ }^{1,2}$ \\ ${ }^{1}$ lcerdanphd@gmail.com (Current Address) \\ ${ }^{2}$ Instituto de Química Física “Rocasolano” (IQFR), Consejo Superior de Investigaciones Científicas \\ (CSIC), Serrano 119, 28006, Madrid, Spain (Affiliation until 2017)
}

S1. Fitting procedure

S2. Performance and sensitivity to noise of VPI method and GUI $\boldsymbol{A g L}$ 


\section{S1. Fitting procedure}

Fitting procedures and recipes are rather crafty. I will present here the general aspects of my recipe, but others could as well be valid. If we wanted to fit eq. (2a) together with eq. (2b) to a set of ASE spectra leaving all parameters free, we would have a huge number of unknowns: $\Omega(\lambda), \gamma(\lambda), \alpha(\lambda), \eta\left(I_{p}\right)$, and those associated to $\Delta n\left(I_{p}\right)$ (eq. (2b)). Such number of unknowns would be generally intractable, and the fitting procedure would probably find a local minimum, not a global one, meaning that the parameters could be unrealistic. Hence a number of constraints have to be included in order to make the fit feasible and its results as trustful and reliable as possible.

To start with, we have seen in eq. (A4h) that $\Omega(\lambda)=\Omega_{0} F(\lambda)$, i.e., it is proportional to the fluorescence spectrum $F(\lambda)$. The fluorescence spectrum can be easily obtained in a spectrofluorimeter, and in fact this is standard procedure when studying new samples and materials. Accordingly, the fluorescence spectrum $F(\lambda)$ is used as a constraint, leaving the proportionality factor $\Omega_{0}$ as a free parameter.

The inspection of eqs. (2a) and (2b) reveals that $\Omega_{0}$ and $\eta$, and $\gamma$ and $\Delta n_{0}$, appear always as a product, i.e., $\Omega_{0} \eta$ and $\gamma \Delta n_{0}$. Whatever the fitting method, in the process of inspecting the parameter space to optimize the fit, an infinite number of combinations would be found such that the product of the fitted parameters is equal to the product of the actual parameters, e.g., $\Omega_{0, \mathrm{fit} 1} \eta_{\mathrm{fit} 1}=\Omega_{0, \mathrm{fit} 2} \eta_{\mathrm{fit} 2}=\ldots=\Omega_{0} \eta$. This leads to a huge uncertainty and makes the determination of errors impossible. To avoid this, I have found convenient to normalize $\Delta n$ and $\eta$ to unity at the maximum pump intensity of the experiment $\left(I_{p, \max }\right)$. Hence, the term $\Delta n_{0}$ in eq. (2b) must be expressed as:

$$
\Delta n_{0}=\frac{1+\frac{I_{p, \max }}{I_{p, 1}^{s}}+\left(\frac{I_{p, \text { max }}}{I_{p, 2}^{s}}\right)^{n}}{\frac{I_{p, \text { max }}}{I_{p, 1}^{s}}+\left(\frac{I_{p, \max }}{I_{p, 2}^{s}}\right)^{m}}
$$

The proportionality factors that are cancelled out in the normalization of $\Delta n$ and $\eta$ are assimilated into $\gamma(\lambda)$ and $\Omega_{0}$, respectively. This has a trade-off in that the fitted $\gamma$ will be, in general, not absolute anymore, but relative. In contrast, the product $\gamma \Delta n$ will be absolute.

In the process of developing this method, I have seen that if $\alpha(\lambda)$ is left as a completely free fitting parameter, the fitted result is in general unreliable, and thus it must be partially constrained. In inspecting eq. (S2a) together with the described behaviour of $\Delta n$ (Figure A5 and Eq. (2b)), it can be seen that in the limit when $I_{p} \rightarrow 0, I_{A S E}\left(L, I_{p}, \lambda\right)$ can be approximated to:

$$
I_{A S E}\left(L, I_{p} \rightarrow 0, \lambda\right) \approx \frac{\Omega(\lambda) \eta\left(I_{p}\right)}{\alpha(\lambda)}\left(1-e^{-\alpha(\lambda) L}\right) \propto \frac{F(\lambda) I_{p}}{\alpha(\lambda)}\left(1-e^{-\alpha(\lambda) L}\right)
$$

In the previous equation the only unknowns are $\alpha(\lambda)$ and a proportionality factor. Thus, fitting eq. (S2) to the measured ASE spectra at pump intensities well-below threshold, one can retrieve an estimation of $\alpha(\lambda)$. This estimation is subsequently refined multiplying it by a parameter $v$ which is determined in the 
subsequent steps of the fit and which value lies close to 1. Finally, $\gamma(\lambda), \eta\left(I_{p}\right)$ and the parameters associated to $\Delta n\left(m, n, I_{p, l}^{s}\right.$ and $\left.I_{p, 2}^{s}\right)$ can be left free.

The fitting procedure to retrieve $\Omega_{0}, \eta, \alpha(\lambda), \gamma(\lambda)$ and the parameters associated to $\Delta n\left(m, n, I_{p, I}^{s}\right.$ and $\left.I_{p, 2}^{s}\right)$ is very sensitive to initial conditions and thus it must be performed in a sequential fashion:

1. $L, I_{p}$, and $\mathrm{F}(\lambda)$ as input parameters. $\alpha(\lambda)$ is obtained fitting eq. (S2) to the ASE spectra at the lowest available pump intensities (well-below threshold). Initial guess: $\alpha(\lambda) \approx 0 \forall \lambda$. Bounds: $\alpha(\lambda) \in[0, \infty) \forall \lambda$.

2. $L, I_{p}, \mathrm{~F}(\lambda)$, and the fitted $\alpha(\lambda)$ as input parameters. $v, \Omega_{0}, \Delta n, \eta$ and $\gamma(\lambda)$ are obtained fitting eq. (2a), together with (2b), to all the available ASE spectra. In this intermediate step, the following constraints are forced into eq. (2b): $m=n$, and $I_{p, 1}^{s}=I_{p, 2}^{s}$. Initial guesses: $v=1, m=2, I_{p, I}^{s}=I_{p}$ for the middle point, $\gamma(\lambda) \propto \alpha(\lambda) / \max (\alpha(\lambda))+I_{A S E}\left(L, I_{p, \max }, \lambda\right) / \max \left(I_{A S E}\left(L, I_{p, \max }, \lambda\right)\right)$. Bounds: $v \in[0, \infty), \Omega_{0} \in$ $[0, \infty), \eta \in[0,1], m \in[1, \infty), I_{p, 1}^{s} \in(0, \infty), \gamma(\lambda) \in[0, \infty) \forall \lambda$

3. $L, I_{p}, \mathrm{~F}(\lambda)$, and the updated $\alpha(\lambda)=v \cdot \alpha(\lambda)$ as input parameters. The definitive $v, \Omega_{0}, \Delta n, \eta$, and $\gamma(\lambda)$ are obtained fitting again eq. (2a), together with (2b) without constraints, to all the available ASE spectra. Initial guesses: $v=1$, previously fitted $\Omega_{0}, \eta, \gamma(\lambda)$ and parameters associated to $\Delta n$. Bounds: the same as in 2, plus $n \in[1, \infty)$ with $n<m$, and $I_{p, 2}^{s} \in(0, \infty)$.

4. (OPTIONAL) Same as 3 , but $\gamma(\lambda) \in(-\infty, \infty) \forall \lambda$

5. Finally, using the fitted $\Delta n, \alpha(\lambda)=v \cdot \alpha(\lambda)$, and $\gamma(\lambda)$, one can compute the ASE threshold solving eq. (6) of the main manuscript.

\section{S2. Performance and sensitivity to noise of VPI method and GUI $\mathbf{A g L}$}

\section{S2.1 Proof of concept with numerical results}

The first step was to assess the performance of the current methodology and software under the ideal and controlled case corresponding to the ASE spectra generated numerically in Appendix A.2 of the main manuscript. Figure S1 recollects the main results corresponding to the fit to the ASE spectra for both pump pulse widths. As can be easily appreciated, the fitting parameters almost mimic to perfection the numerical results. The ASE threshold, computed from the fitting parameters using eq. (6) and represented in figure S1a as vertical lines, lays at the pump energy where the peak intensity stops growing linearly (fluorescence) and starts to grow superlinearly (ASE). One could expect to find the threshold where there is a clearer change in $\mathrm{I}_{\mathrm{ASE}}$ slope. But a closer inspection to the dependency on the pump energy of the net optical gain $g=\gamma \Delta n-\alpha$ at the ASE peak (figure S1b), reveals that the threshold lays in the proximity to the pump energy at which $g$ $=0$. The exact point varies from sample to sample and will ultimately depend on the particular values of the gross gain $\gamma$, the total losses $\alpha$, and the exact shape of $\Delta n$ (eq. (6)). 

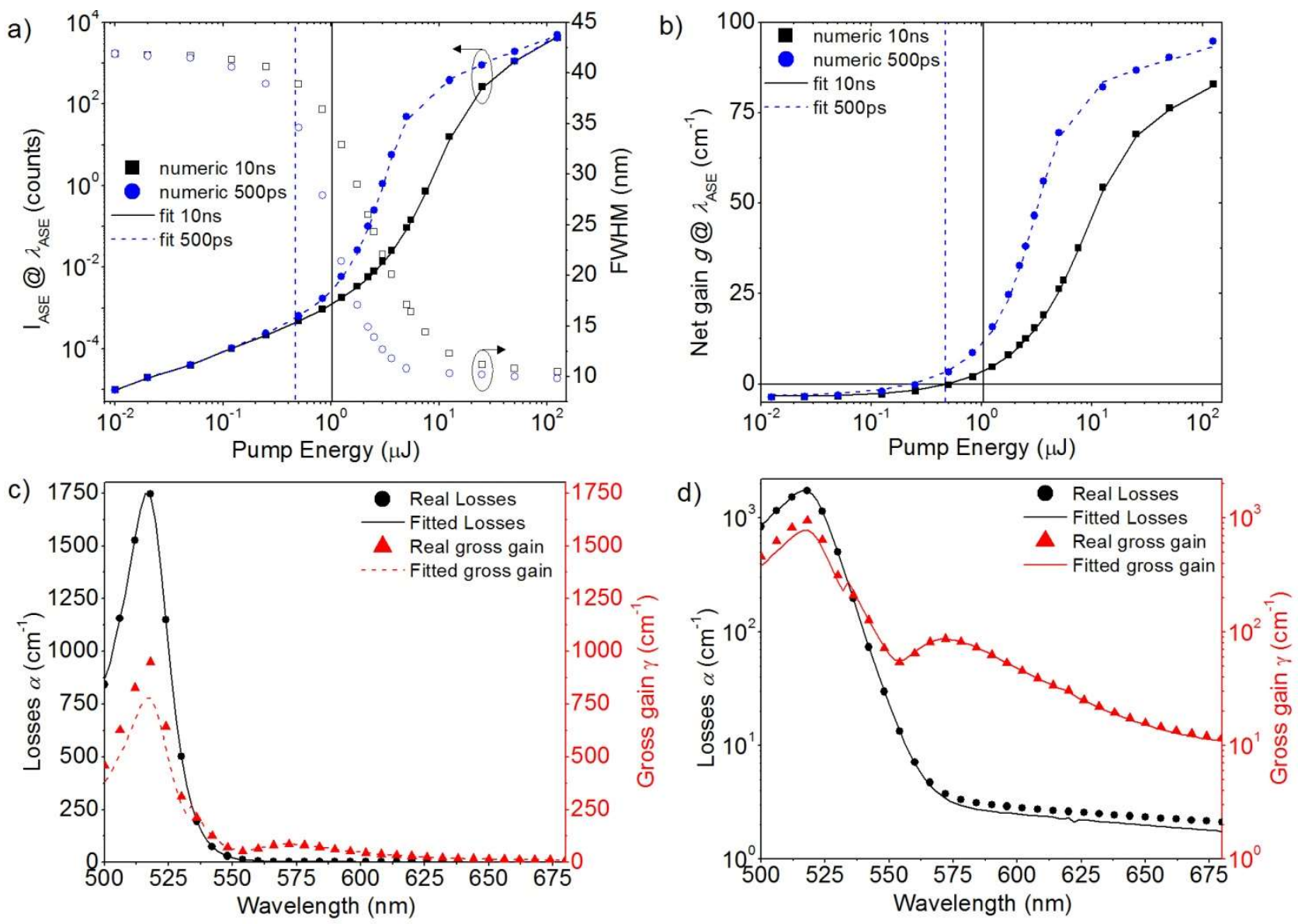

Figure S1: Numerical vs. fitted values: a) ASE intensity at the peak wavelength $(572 \mathrm{~nm})$ and ASE full width half maximum (FWHM) as a function of pump energy. b) Net optical gain $g=\gamma\left(\lambda_{\mathrm{ASE}}\right) \Delta n\left(I_{p, t h}\right)-\alpha\left(\lambda_{\mathrm{ASE}}\right)$ at peak wavelength as a function of pump energy. c) Losses and gross gain spectra. d) The same as c) but in semilog-y scale. The vertical solid and dashed lines in a) and b) mark the position of the ASE threshold calculated with eq. (6) for, respectively, $\tau_{\mathrm{p}}=10 \mathrm{~ns}$ and $\tau_{\mathrm{p}}=500 \mathrm{ps}$. In all plots, the dots represent the values obtained numerically and the lines show the best fit of eqs. (2a) and (2b) to the numerically generated ASE spectra.

The fitted losses and gross gain spectra are highly accurate (Figure S1c and S1d), presenting slight differences with respect to the real spectra at, respectively, the long and short wavelength spectral wings of the ASE spectra (cf. figure A2b). These differences are not due to an inaccuracy in the method. They are a direct consequence of the numerical precision of the software, which struggles to accurately determine the correct parameters in regions where the signal and relative changes are close to, or smaller than, the decimal precision. Nevertheless, this error barely affects the overall fit and it will in fact be out-weighted by the error associated to the inherent noise at the spectral wings in experimental ASE spectra. In any case, it is important to note and remember that the fit will be much more precise in the vicinity of the ASE peak. In particular, at the ASE peak $(572 \mathrm{~nm})$ the real losses and gross gain are, respectively, $3.7 \mathrm{~cm}^{-1}$ and $89.2 \mathrm{~cm}^{-1}$, whereas the fitted values amount to $3.4 \mathrm{~cm}^{-1}$ and $85.9 \mathrm{~cm}^{-1}$. 


\section{S2.2 Sensitivity to experimental noise}

In figure S1 it was shown that the fitting parameters almost mimicked to perfection the numerical results. This does not come as a surprise, since the fitted spectra were ideal cases in which the noise was null. To check the influence of the experimental noise in the fitting parameters retrieved from the current methodology and the GUI $A g L$, a certain level of random noise was artificially superimposed onto the numerically generated ASE spectra. Each spectrum $I_{A S E}\left(I_{p}, \lambda\right)$ (figure S2a) was modified as follows: 1) An off-set error is added to the original spectrum: $I_{\text {noise, }}\left(I_{p}, \lambda\right)=I_{A S E}\left(I_{p}, \lambda\right)+\operatorname{noise}\left(\sigma, I_{p}\right) \cdot \min \left(I_{A S E}\left(I_{p}, \lambda\right)\right)$. 2) To that spectrum, an overall intensity error is added: $I_{\text {noise, } 2}\left(I_{p}, \lambda\right)=\operatorname{noise}\left(\sigma, I_{p}\right) \cdot I_{\text {noise },}\left(I_{p}, \lambda\right)$. 3) To that spectrum, an spectral noise is added: $I_{A S E, \text { noise }}\left(I_{p}, \lambda\right)=\operatorname{noise}(\sigma, \lambda) \cdot I_{\text {noise, } 2}\left(I_{p}, \lambda\right)$. Finally, the pump values are as well treated with noise $\left(I_{p \text {,nise }}=\operatorname{noise}\left(\sigma / 5, I_{p}\right) \cdot I_{p}\right)$. The function noise $(\sigma, \mathrm{x})$ is a vector the same size of $\mathrm{x}\left(I_{p}\right.$ or $\left.\lambda\right)$ with random numbers extracted from a normal distribution centred at 1 with standard deviation $\sigma$. Every time this function is applied, a new pool of random numbers is used. Figure S2b shows an example of the resulting ASE spectra when assuming a 30\% error $(\sigma=0.3)$, the maximum considered for this analysis. The noisy ASE spectra $I_{A S E \text {,noise }}\left(I_{p}, \lambda\right)$ and pump values $I_{p, \text { noise, }}$, together with the fluorescence spectrum, are fed into the software $\mathrm{AgL}$ and the fit is performed. For each noise level, ten different sets of modified ASE spectra and pump values were analyzed.

Irrespective of the noise level, the GUI $A g L$ has no problem in fitting the experimental values. Nevertheless, the influence of noise in the fitting parameters is evident, overall when looking at the losses and gross gain spectra (Figure S2c). Note that the former is more sensitive to noise than the latter. As was explained in the fitting procedure supplementary section S1, to fit the losses only the ASE spectra corresponding to the lowest pump values are used ( 3 in this case) to avoid the presence of amplification, whereas the gross gains are obtained using all of them (20 in this fit). The higher the number of spectra used for the fit, the smoother the fitting parameters become. This explains why the gross gain spectrum is less noisy that the losses spectrum.

Anyhow, the fitting parameters in the presence of noise are still reasonably similar to the ones without noise. To have a clearer insight into the differences, one must resort to evaluating the influence of the noise level on the gross gain, the losses, and the ASE threshold (Figure S2d). When increasing the noise level, both the gross gain and the losses increase consistently. Interestingly, it seems that both parameters increase by the same amount (less than $2 \mathrm{~cm}^{-1}$ ), in such a way that the net optical gain $g=\gamma \Delta n-\alpha$ at the ASE peak remains almost unaffected by noise (Figure S2e). This immunity to noise is experienced as well by the ASE threshold, which remains almost constant, differing by less than a $10 \%$ from the values without noise (Figure S2d). The previous analysis bears witness of the robustness against noise and reliability of the presented methodology and the developed software. 

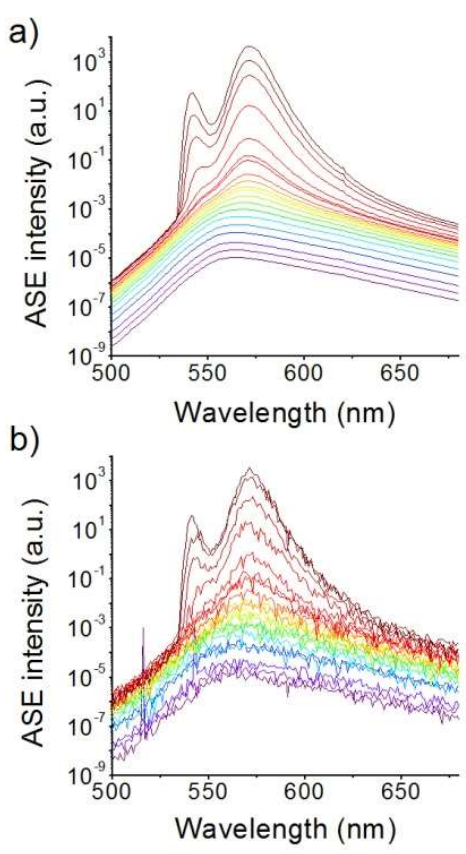

c)

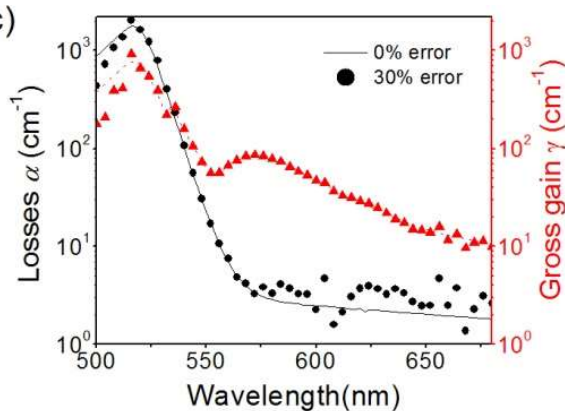

e)

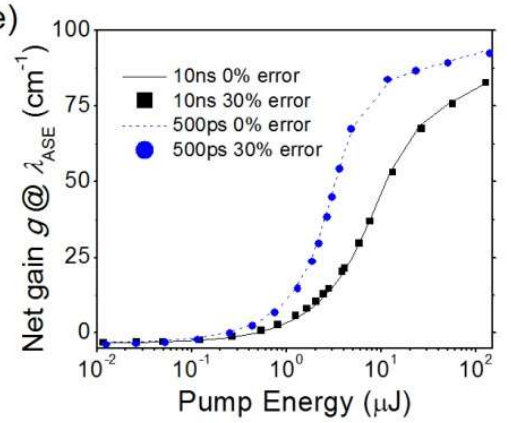

d)

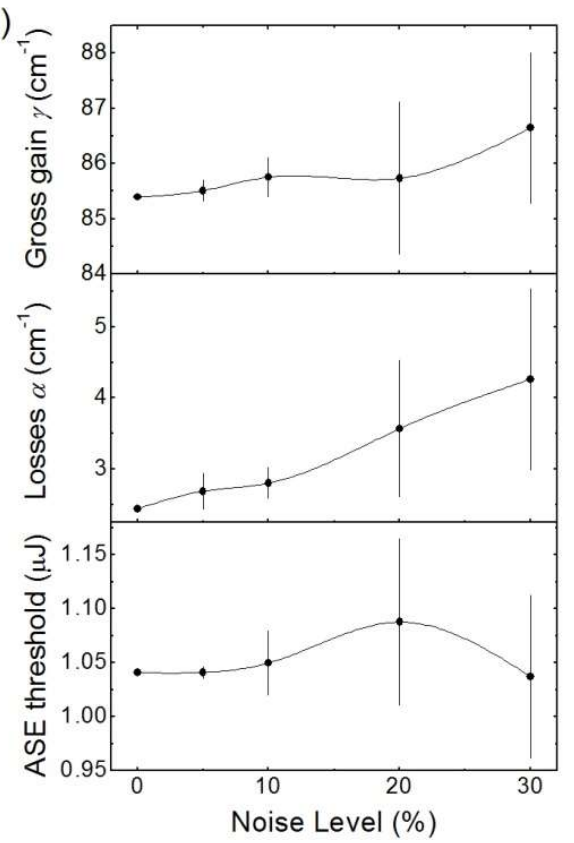

Figure S2: Numerically generated ASE spectra before (a) and after (b) noise treatment $(\sigma=0.3)$. c) Comparison of fitted losses and gross gain spectra obtained from clean (lines) and noisy (dots) ASE spectra. d) Influence of noise level on fitted losses and gross gain at ASE peak, and ASE threshold. Each point and error bar represents the average and standard deviation, respectively, of the fitting parameters obtained over 10 sets of measurements. e) Comparison of fitted net optical gain $g$ at ASE peak obtained from clean (lines) and noisy (dots) ASE spectra. 